\title{
Massive MIMO, mmWave and mmWave-Massive MIMO Communications: Performance Assessment with Beamforming Techniques
}

Tewelgn Kebede Engda ( $\nabla$ tewelgn@gmail.com )

Addis Ababa Institute of Technology, Addis Ababa University and Kempten University of Applied

Sciences https://orcid.org/0000-0002-6494-2609

Yihenew Wondie

Addis Ababa University, Institute of Technology

Johannes Steinbrunn

Hochschule Kempten

\section{Research}

Keywords: Analog beamforming, digital precoding, hybrid analog-digital beamforming, Massive MIMO, mmWave, mmWave-massive MIMO

Posted Date: September 2nd, 2020

DOI: https://doi.org/10.21203/rs.3.rs-69959/v1

License: (c) (i) This work is licensed under a Creative Commons Attribution 4.0 International License. Read Full License 


\title{
RESEARCH
}

\section{Massive MIMO, mmWave and mmWave-Massive MIMO Communications: Performance Assessment with Beamforming Techniques}

Tewelgn Kebede Engda ${ }^{*}$, Yihenew Wondie ${ }^{2}$ and Johannes Steinbrunn ${ }^{3}$

\begin{abstract}
A considerable amount of enabling technologies are being explored in the era of fifth generation (5G) mobile system. The dream is to build a wireless network that substantially improves the existing mobile networks in all performance metrics. To address this $5 \mathrm{G}$ design targets, massive MIMO (multiple input multiple output) and mmWave (millimeter wave) communication are also candidate technologies. Luckily, in many respects these two technologies share a symbiotic integration. Accordingly, a logical step is to integrate mmWave communications and massive MIMO to form mmWave-massive MIMO which substantially increases user throughput, improve spectral and energy efficiencies, increase the capacity of mobile networks and achieve high multiplexing gains. Thus, this work analyses the concepts, performances, comparison and discussion of these technologies called: massive MIMO, mmWave Communications and mmWave-massive MIMO systems jointly. Besides, outcomes of extensive researches, emerging trends together with their respective benefits, challenges, proposed solutions and their comparative analysis is addressed. The performance of hybrid analog-digital beamforming architecture with a fully digital and analog beamforming techniques are also analyzed. Analytical and simulation results show that the low-complexity hybrid analog-digital precoding achieves all round comparable precoding gains for mmWave-Massive MIMO technology.
\end{abstract}

Keywords: Analog beamforming; digital precoding; hybrid analog-digital beamforming; Massive MIMO; mmWave; mmWave-massive MIMO

\section{Introduction}

To help the rapid growth of current mobile traffic and the development of modern wireless applications and services, developers and regulatory bodies worldwide have planned to develop 5G wireless networks [1]. Hence, research and development of $5 \mathrm{G}$ standards discloses a range of critical innovations that are expected to meet the enthusiastic system requirements. A few of the specific performance requirements for $5 \mathrm{G}$ cellular networks are [2]: high data rates, reduced latency, low energy consumption, high scalability, high connectivity and security. Accordingly, the key interdependent technologies to address $5 \mathrm{G}$ design requirements are [3-5]: Ultra-dense Networks (UDNs), millimeter Wave (mmWave) Communications and Massive MIMO (Multiple Input Multiple Output). Individually, each of these innovations would give an order of magnitude increase in the efficiency of wireless

\footnotetext{
${ }^{*}$ Correspondence: tewelgn@gmail.com

${ }^{1}$ Addis Ababa Institute of Technology, Addis Ababa University, Addis Ababa, Ethiopia

Full list of author information is available at the end of the article
}

systems. Fortunately those three approaches share a mutualistic convergence in many respects [6]. That is, the very short wavelength of mmWave frequencies is attractive for massive MIMO since the physical size of the antenna arrays can be considerably reduced, smaller cell sizes are promising for mmWave shortrange communications, while the large antenna gains offered by massive MIMO are effective in alleviating the sever path loss of mmWave signals. So, if there is a rational way to integrate these three approaches, then one would aim to accomplish the 1000-fold improvement in 5 G efficiency. Stepping in this direction, we already have mmWave technology that takes the main design schamtics of MIMO technology and moves up the operating frequency to the mmWave band (Fig. 1). The combination of mmWave and massive MIMO will therefore be a natural step in harnessing the attributes of large area coverage and localized small cell hotspots, leading to the phrase "mmWave-massive MIMO".

MmWave-massive MIMO is a promising technology for future cellular systems that transmits gigabits per 
second by leveraging the large bandwidth available at mmwave frequencies. The path loss at mmWave frequencies makes it difficult to implement these systems, however large antenna arrays can be packed into small chips at mmwave frequencies which provides a reasonably strong received signal. Similarly, large antenna arrays help the design of beamforming techniques to direct the signal in a certain direction, hence reducing the path loss problem [7]. Even if mmWave-massive MIMO has attractive attributes, it still has challenges such as large number of antennas that eventually introduce very high or even unrealistic complexity of hardware and power consumption [8].

In this performance evaluation of mmWave-Massive MIMO technology, we attempt a holistic overview of concepts, comparison and discussion of three $5 \mathrm{G}$ candidate technologies called massive MIMO, mmWave Communications and mmWave-massive MIMO systems jointly. The aim is to describe in detail the theory behind every associated technique with the state-ofthe-art methods being explored for these three technologies. It, therefore, serves to address mmWavemassive MIMO and its interrelationship with each other and existing technologies, with special attention on the areas of precoding and recent developments. Therefore, to the authors' best knowledge, there has been no performance evaluation available in the scientific arena which has discussed massive MIMO, mmWave communications and mmWaveMassive MIMO technology jointly, although there are quite a few surveys available such as [9], which deliberated these three technologies separately. Hence, to achieve $5 \mathrm{G}$ goals, these three candidate technologies should be studied together. This paper, therefore, presents a comprehensive overview, comparison, discussion and analyses of massive MIMO, mmWave Communication and mmWave-massive MIMO communication system with aim to explore the emerging trends, challenges and proposed approaches for mmWave-massive MIMO system in the march towards $5 \mathrm{G}$ era.

The remaining contents of the paper is arranged as follows. We briefly summarize the existing works in the context of our work in Section 1 and massive MIMO is described with detail descriptions in Setion 2. mmWave communications, mmWave-Massive MIMO and Beamforming with their system and channel are desribed in Sections 3, 4 and 5 respectively. We evaluate the performance of our solution scheme in Section 6. Finally, Section 7 concludes the paper with brief direction of future research direction.

\section{Massive MIMO Communication Technology}

Massive MIMO, which is also called full dimension MIMO, is a viable system performance optimization technology. This encompasses the use of antenna arrays with numerous antenna elements for large-scale application of high-gain adaptive beamforming and multi-user spatial multiplexing. Massive MIMO offers high data rates to many users in dense deployment scenarios [10]. For 5G higher frequency band communication systems such as mmWaves, large-scale antenna arrays would be a system pre-requisite for combating the poor propagation characteristics within these bands. This technology also enhances spectral and energy efficiency by adding the number of antennas at the BS (Basestation) to serve users with directed signal and reasonably simple signal processing [11]. Accordingly, massive MIMO has potential advantages like: capacity and link reliability, high spectral and energy efficiency, security enhancement and robustness improvement, high degree of freedom, cost efficiency and simple signal processing [12-16]. Similarly, massive MIMO has also few key features:

i. Linear Signal Processing: Massive MIMO contains a large number of BS antennas and the signal processing is with matrices and/or vectors of quite large dimensions. Hence a simple signal processing method is desired. In this regard, the linear signal processors (linear uplink detectors and downlink precoders) perform near optimal [17].

i) Time Division Duplexing (TDD): The signaling overhead for channel estimation is proportional to the number of BS antennas. In contrast, for TDD, the signaling overhead for channel estimation is not dependent on BS antennas [14]. Therefore, implementing a TDD operation is preferred .

ii) Favorable Propagation: In many propagation environments, the use of an large number of BS antennas over the number of users produces a favorable propagation where the channel vectors between the users and the BS are pairwise (nearly) orthogonal. The effect of inter-user interference and noise can be minimized by simply linear signal processing under favorable propagation [18].

iii) Channel Hardening: Refers to the phenomenon where the massive MIMO channel matrix approaches their expected values, when the number of antennas approaches infinity. That is as the size of channel gain matrix rises, the off-diagonal elements of the matrix become weaker compared to the diagonal terms [15], which approaches the effective channel deterministic. Under these conditions, the channel becomes (nearly) deterministic and hence, the effect of 
small-scale fading is averaged out. The system scheduling, power control, etc., can be done over the largescale fading time scale instead of over the small-scale fading time scale since this effectively removes the effects of fast fading. This effectively simplifies the signal processing significantly.

iv) Linear Signal Processing: The use of large number of antennas in massive MIMO yields favorable propagation and channel hardening which really simplifies the signal processing in mmassive systems [17].

v) Physical Size: FFor massive MIMO systems, when we talk about large BS antenna array, it does not necessarily means that it must be large physically. Rather, it is a collection of small physical size, low power and low cost antennas [19].

vi) Scalability: The time for estimating a channel is uncorrelated to the number of BS antennas. Hence, even if we keep on increasing the number of BS antennas there will be no increase in signaling overhead for the channel estimation. In addition, the linear signal processing is simple for each user and is independent of other users, (no multiplexing or demultiplexing is performed at the users) increasing or decreasing the number of served users does not affect other users.

Massive MIMO technology, does not have only merits. As any other new technologies, faces challenges. To make this technology reach the efficient implementation stage, the challenges that need further study and investigation are: channel estimation, pilot contamination, antenna array design, hardware impairment, precoding and detection [20-22].

\subsection{Massive MIMO Precoding}

Precoding is a significant signal processing solution that uses CSI at the transmitter to optimize link performance. To concentrate the spatial data streams at the users direction, the BS has to precode downlink data. That is, precoding at the transmitter is used in the downlink to concentrate each signal to its intended user to cancel interference. If the interference cancellation is not done properly, the desired output is not obtained at the receiver. Hence, precoding techniques are used to coordinate the transmission of data [23]. Basically, precoding techniques are divided into linear and non linear. The linear precoding methods are computationally simpler than their nonlinear counterparts. On the other hand, nonlinear precoding schemes can outperform the linear precoding schemes, at the cost of significantly high computational complexity. However, with an increase in the number of antennas at the BS, linear precoders are shown to be near-optimal [24]. Therefore, it is more practical to use low-complexity linear precoding techniques in massive MIMO systems. Linear precoding techniques are: zero forcing
(ZF), matched filter (MF), minimum mean square error (MMSE) precoding, truncated polynomial expansion (TPE) and phased zero forcing (PZF). By contrast non-linear precoders are: the optimal Dirty Paper Coding, the near optimal Tomlinson-Harashima precoding and Vector Perturbation Precoding. Readers could refer to [25], for detail of these linear precoding techniqus.

\subsection{Massive MIMO System Model}

Consider a single-cell downlink massive MIMO system, where the BS is equipped with $M$ antennas to support $K$ single-antenna users in the same time-frequency resource as shown in Figure 2.

It is also considered that the channel is a Rayleigh fading and ergodic with perfect CSI at the transmitter. If $\mathbf{x}$ denotes the complex valued $M \times 1$ transmitted signal vector from the $M$ antennas then the $K \times 1$ received signal vector $\mathbf{y}$ at the users is given by [6]

$$
\mathbf{y}=\sqrt{\mathrm{p}_{d}} \mathbf{H x}+\mathbf{n}
$$

where $\mathbf{H} \in \mathbb{C}^{K \times M}$ is a channel matrix between the BS antennas $M$ and $K$ users. The elements of $\mathbf{H}$ are considered to be i.i.d (independent and identically distributed) complex Gaussian random variables with mean zero and unit variance. The signal received by the $k^{\text {th }}$ user after using the linear precoding scheme is given by $\mathbf{x}=\mathbf{W} \mathbf{s}$ which is the precoded signal at the BS, $\mathbf{s} \in \mathbb{C}^{K \times 1}$ is the information bearing signal with $\mathbb{E}\left\{\mathbf{s s}^{H}\right\}=\mathbf{I}_{K}, \mathbf{W} \in \mathbb{C}^{M \times K}$ is the precoding matrix at the BS and $p_{d}$ is the downlink transmit power for user $k . \mathbf{n}$ is AWGN (additive white Gaussian noise) vector at the users with unit variance and mean zero elements. Then, signal received by the $k^{t h}$ user after using the linear precoding scheme is given by [26]

$$
y_{k}=\sqrt{p_{d}} \mathbf{h}_{k} \mathbf{w}_{k} s_{k}+\sum_{i=1, i \neq k}^{K} \sqrt{p_{d}} \mathbf{h}_{k} \mathbf{w}_{i} s_{i}+n_{k}
$$

where $\sqrt{p_{d}} \mathbf{h}_{k} \mathbf{w}_{k} s_{k}$ is the desired signal for user $k$ and $\sum_{i=1, i \neq k}^{K} \sqrt{p_{d}} \mathbf{h}_{k} \mathbf{w}_{i} s_{i}$ is the multi-user interference signal. The SINR (signal-to-interference-plus-noise-ratio) of user $k$ is

$$
\mathrm{SINR}_{k}=\frac{p_{d}\left|\mathbf{h}_{k} \mathbf{w}_{k}\right|^{2}}{p_{d} \sum_{i=1, i \neq k}^{K}\left|\mathbf{h}_{k} \mathbf{w}_{i}\right|^{2}+1}
$$

that is a function of the transmit precoding vector $\mathbf{w}_{k}$ and the channel $\mathbf{h}_{k}$. The respective models of linear precoding techniques are expressed as follows. The detail of these models is found in [25]. 


$$
W= \begin{cases}H^{H}, & \mathrm{MF} \\ H^{H}\left(H H^{H}\right)^{-1}, & \mathrm{ZF} \\ \beta\left(H H^{H}+\alpha I_{K}\right)^{-1} H^{H}, & \mathrm{MMSE} \\ \sum_{j=0}^{j-1} w_{j}\left(H^{T} H^{*}\right)^{j} H^{T}, & \mathrm{TPE} \\ H_{e q}^{H}\left(H_{e q} H_{e q}^{H}\right)^{-1} \lambda, & \mathrm{PZF}\end{cases}
$$

where $\beta$ is the power normalization parameter, $\alpha=$ $K / P_{d}$ is the regularization factor to be optimized, $w_{j}$ is the coefficient of the precoder polynomial of order $J$ and $\lambda \in \mathbb{R}^{K \times K}$ is a positive diagonal matrix for column power normalization. Considering the data transmission in favorable propagation conditions, the performance of massive MIMO can be evaluated using simple linear signal processing techniques. Their numerical results are evaluated in terms of SNR (signal to noise ratio), sum rate and energy efficiency [27].

Figure 3 illustrates the achievable sum rate of massive MIMO performance using various linear processing techniques with respect to basestation antennas. As clearly observed in the figure, the achievable sum rate increases exponentially as the number of antenna elements increases in the BS. On the other hand, as the number of BS antennas increases, the spectral efficiency with linear signal processing is almost the same with Shannon sum capacity achieved by optimal precoders.

The simulation result in Fig.4 shows the sum rate versus SNR for ZF, RZF and MRT with $M=64$ and $K=8$. The signal to noise ratio varies from $-10 d B$ to $25 d B$. MRT has better bandwidth efficiency than $\mathrm{ZF}$ in the low SNR regime, and vice versa in the high SNR regime.

The result also indicates that when the transmit power is a minimal state, the MRT precoding scheme is a reasonable option. On the contrary, the sum rate with $\mathrm{ZF}$ and RZF precoding schemes attain a higher rate than with MRT at the high SNR level. As the SNR increases, the total rate with ZF and RZF precoding schemes increases exponentially as these precoding strategies promote inter-user-interference and noise cancelation. RZF's achieveable rate performs specifically better over the full spectrum of SNRs than ZF and MRT.

\section{Millimeter-Wave Communications}

The primary focus of $5 \mathrm{G}$ systems are to increase the data rate by a factor of 1,000 compared with the previous 4G [5]. A fairly direct approach to improve the data rate is to increase the bandwidth used for transmission. Nonetheless, the improvements in bandwidth which can be gained by using the typically used sub-6 $\mathrm{GHz}$ frequency bands effectively are very low. Correspondingly, turning to frequency bands that have seen minimal use or no use up to now is reasonable. In this respect, the mmWave frequency band [28] is the extremely high frequency range from 30-300 GHz electromagnetic spectrum, with the corresponding wavelength of 1-10 mm. The key propagation-related barriers in the realization of mmWave cellular are: the freespace path loss, scattering and the non-line-of-sight (NLoS) paths are weaker making blockage and coverage holes more noticeable [29]. In addition, due to the usage of larger bandwidth channels, the noise power is larger. In order to achieve a high SNR reliably across a cell, mmWave networks must utilize high gain electronically steerable directional antennas. That is, it must beamform or precode data on large antenna arrays. However, due to the small wavelength, arrays of realistic dimensions will actually house orders of magnitude and provide sufficient array gain to overcome path loss and ensure high SNR at the receiver. In addition to using adaptive steerable arrays to overcome blockages , mmWave networks can also be made very dense to benefit from the current trend pushing cellular system to a higher heterogenerous network containing relays and small cells.

In the past few years, the general attitude toward using mmWave frequencies for communications has dramatically changed because of two reasons [30]. First, taking into account the trend towards a reduction in cell sizes and the emergence of short-range techniques such as D2D communication, the required transmission distances have been significantly reduced. Second, robust programs at measuring platforms have shown that the loss of the route is not as serious as was previously believed. In essence, the use of large antenna arrays will offset largely the less favorable conditions of propagation compared to lower frequencies. One remaining challenge is the strong blockage of mmWave signals, which prevents building walls from penetrating and renders a line of sight ( LOS) necessary for secure communication.

Compared with current wireless technologies, the special features of mmWave communications are: extremely wide bandwidth and capacity, low probabilityof-intercept, small element sizes, narrow beams and directivity [28,31].

Given the huge bandwidth capacity, mmWave signal transmissions suffer from fundamental technical challenges such as intense path loss, high penetration loss, high power consumption, blockage due to shadowing, hardware impairment, etc [32]. 
Operating in the mmWave bands is particularly challenging because of the poor road-loss conditions within these bands. The free-space reference-distance pathloss $\left(P_{L}\right)$ according to [33] can be expressed as:

$$
\mathbf{P}_{L}[d B](\mathbf{d})=20 \log \left(\frac{4 \pi \mathbf{d}_{o}}{\lambda}\right)+10 \mathbf{n} \log \left(\frac{\mathbf{d}}{\mathbf{d}_{o}}\right)+\mathbf{X}
$$

where, $d_{o}$ is the reference distance, $d$ is the transmitterreceiver separation distance in meters, $n$ is the pathloss exponent and $X$ is a shadow fading term that is a zero-mean Gaussian variable with a specified standard deviation. According to the above free-space path-loss model, the difference between today's cellular systems and the mmWave systems is the result of the difference in the wavelength that shows up in the denominator of the first term of the path-loss equation.

Key to operating in the mmWave bands will be the use of large-scale antenna arrays to overcome the poor path-loss situation in those bands. Luckily, the antenna array size is determined by the size of individual antenna elements which is influenced by the center frequency wavelength at which the device operates. As the carrier frequency increases, the size of the antenna array will correspondingly decrease, so that it will be feasible from a form-factor point of view to have mmWave antenna arrays with hundreds of elements [32].

From this Fig. 5, it can be observed that in some special bands such as $35 \mathrm{GHz}, 94 \mathrm{GHz}, 140 \mathrm{GHz}$, and $220 \mathrm{GHz}, \mathrm{mm}$ Wave propagation experiences relatively small attenuation. Therefore, in these mmWave bands, long distance communication can be realized which is well suitable for peer to peer communication. However, in the $60 \mathrm{GHz}, 120 \mathrm{GHz}, 180 \mathrm{GHz}$ bands, mmWave signals attenuate severely as high as $15 \mathrm{~dB} / \mathrm{km}$, which are known as "attenuation peak" [28]. Meanwhile, mmWave signals will experience poor diffraction when encountering blockages owing to the short wavelengths. This effect significantly shorten the transmission range of mmWave signals and easily bring mmWave links to the disconnected state. Thanks to the rapid progress in RF integrated circuits, beamforming based on large-scale mmWave antenna arrays has been widely exploited to extend the coverage of mmWave networks.

\subsection{System and Channel Model}

\section{A. System Model}

Consider the downlink multiuser mmWave MIMO system with a $B S$ serving $K$ users simultaneously as shown in Fig. 6 . The base station is equipped with $N_{R F}$
$R F$ chains and $N_{t}$ transmit antennas. Each mobile station (MS) is equipped with $N_{r}$ receiving antennas. The base station communicates with each $M S$ via a single stream. Thus, the total number of streams transmitted is $N_{S}=U$. Besides, the number of MS does not exceed the number of basestation $R F$ chains i.e., $N_{S} \leq N_{R F}$. Perfect knowledge of the CSI is assumed at the $B S$. The precoder at the $B S$ is a hybrid precoder which is decomposed into the analog precoder and baseband precoder.

The spatial multiplexing gain of hybrid precoding is limited by $N_{R F} \leq N_{t}$. The basestation applies an $N_{R F} \times N_{S}$ digital precoding matrix $D_{B B}=$ $\left[d_{B B}^{1}, d_{B B}^{2}, \ldots, f_{B B}^{k}\right]$ where $f_{B B}^{k}(k \in[1,2, \ldots, K])$ is an $N_{R F} \times 1$ vector, followed by an $N_{t} \times N_{R F} R F$ precoding matrix. The analog precoding matrix $\left(A_{R F}\right)$ is used as analog phase shifter and its parameters are constant modules that can be standardized to fulfill $\left|A_{R F}[i, j]\right|=\frac{1}{\sqrt{N_{t}}} \forall_{i}=1,2, \ldots, N_{t}, j=1,2, \ldots, N_{R F}$ which is is the $(i, j)^{t h}$ element of analog precoding matrix. Therefore, the transmitting signal is

$$
\mathbf{x}=\mathbf{A}_{R F} \mathbf{D}_{B B} \mathbf{s}
$$

where $\mathbf{s}=\left[s_{1}, s_{2}, \ldots, s_{k}\right]^{T}$ is the message to be transmitted $\left(N_{s} \times 1(k \times 1)\right)$ vector and normalized as $\mathbb{E}\left[\mathbf{s s}^{H}\right]=\frac{1}{\left(N_{s}\right)} \mathbf{I}_{k}$. The total power constraint of the hybrid precoding matrix is enforced by normalizing $D_{B B}$ as $\left\|D_{B B} A_{R F}\right\|^{2}=N_{S}$.

The received signal $y_{k}$ observed by the $k^{t h} M S$ is [35]

$$
y_{k}=\sqrt{\rho} \mathbf{H}_{k} \sum_{n=1}^{K} \mathbf{A}_{R F} \mathbf{D}_{B B} \mathbf{s}_{n}+\mathbf{n}_{k}
$$

where $\mathbf{H}_{k} \in \mathbb{C}^{N_{r} \times N_{t}}$ matrix which indicates the channel between the $B S$ and the $k^{t h}$ user and $n_{k} \in$ $\mathcal{C N}\left(0, \sigma^{2}\right)$ represents the Complex Gaussian noise vector and $\rho$ represents the average received power.

\section{B. mmWave Channel Model}

Millimeter-wave channels are expected to have limited scattering. Every scatterer is anticipated to contribute a single path of propagation between the transmitter and receiver [36]. An extended SalehValenzuela model is commonly applied in mmWavemassive MIMO channel modeling. Thus, our research work considers this model due to the high antenna correlation and the limited spatial selectivity [37]. The hypothesis in which each scatter represents a single path is assumed. An adoption of a geometric channel 
model with $L_{k}$ scatters is used for the channel of the $k^{t h}$ user. In this model, the $k^{\text {th }}$ user channel matrix $H_{k} \in \mathbb{C}^{N_{r} \times N_{t}}$ can be expressed as

$$
\mathbf{H}_{k}=\sqrt{\frac{N_{t} N_{r}}{L_{k}}} \sum_{l=1}^{L_{k}} \alpha_{k, l} \mathbf{a}_{k, r}\left(\theta_{k, l}\right) \mathbf{a}_{k, t}^{H}\left(\phi_{k, l}\right)
$$

where $\alpha_{k, l}$ is the complex gain of the $l^{t h}$ path, which includes the path loss with $\mathbb{E}\left|\alpha_{k, l}\right|^{2}=\beta, \beta$ is a normalization constant. The variables $\phi_{l} \in[0,2 \pi]$ and $\theta_{l} \in[0,2 \pi]$ are the $l^{t h}$ path's azimuth angles of departure or arrival $(A o D s / A o A s)$ of the transmitter and receiver respectively. $\mathbf{a}_{k, t}\left(\phi_{k, l}\right)$ is the antenna array response vector of the $B S, \mathbf{a}_{k, r}\left(\theta_{k, l}\right)$ is the antenna array response vector of the $k^{t h} M S, L_{k}$ is the number of propagation paths for the channel of the $k^{t h} M S$ with $l \in\left[1,2, \ldots, L_{k}\right]$. We assume the base station and mobile station get knowledge about the structure of their antenna arrays. It is assumed that the base station and mobile station get knowledge about the structure of their antenna arrays. With respect to structure, antenna arrays are classified as either a uniform linear array (ULA) or a uniform planar array (UPA) (Fig.7). But, for mmWave-massive MIMO channels, UPAs are much more appropriate since they yield smaller antenna array dimensions. This encourages the packing of more antenna elements in a acceptable-sized array that allows beamforming in the elevation domain (3D beamforming) [4].

In the case of $U P A s, N=W_{1} W_{2}$ where $W_{1}$ and $W_{2}$ represent the number of antenna elements on the horizontal and vertical, respectively, with $0 \leq x \leq W_{1}-1$ and $0 \leq y \leq W_{2}-1$. If a ULA is assumed, the array response vectors at the basestation or transmitter, $\mathbf{a}_{t}(\phi)_{l}$ is defined as

$\mathbf{a}_{t}(\phi)_{l}=\frac{1}{\sqrt{N}}\left[1, e^{j\left(\frac{2 \pi}{\lambda}\right) d \sin \left(\phi_{l}\right)}, \ldots, e^{j\left(N_{t}-1\right)\left(\frac{2 \pi}{\lambda}\right) d \sin \left(\phi_{l}\right)}\right]^{T}$

The receiver's array response vectors is written in the same way.

$$
\begin{aligned}
& \mathbf{a}_{r}\left(\theta_{l}\right)=\frac{1}{\sqrt{N}}\left[1, e^{j \frac{2 \pi}{\lambda} d(x \sin (\phi) \sin (\theta)+y \cos (\theta))}, \ldots\right. \\
& \left.e^{j \frac{\pi}{\lambda} d\left(\left(W_{1}-1\right) \sin (\phi) \sin (\theta)+\left(W_{2}-1\right) \cos (\theta)\right)}\right]^{T}
\end{aligned}
$$

where $\lambda$ denotes the wavelength, $d$ is the spacing between two antennas (typically, $d=\frac{\lambda}{2}$ ), $\phi$ is the azimuth angle, $\theta$ is the elevation angle and $N$ is antenna elements number.

\section{4 mmWave-Massive MIMO Communications}

The mmWave and massive MIMO technologies can be combined together to meet the demands of future $5 \mathrm{G}$ networks. This technological combination of massive MIMO and mmWave systems have given birth to mmWave-massive MIMO [38], which offers a broad spectrum of advantages: (a) availability of multiplexing and array gains because of large number of transmit and receive antennas, (b) extreme data rates due to the wide bandwidth that is found at millimeter frequencies and (c) reduced interference due to narrow beamforming. Using the joint functionalities of the huge available bandwidth in the mmWave frequency bands and high multiplexing gains with massive antenna arrays, mmWave massive MIMO has the potential to enhance user throughput, boost mobile network capacity, raise spectral and energy efficiency [39].

MmWave-massive MIMO springs as a technology that integrates potentials of the huge available mmWave bandwidth and the high gains of massive MIMO antenna arrays. When using mmWave-massive MIMO in the field of HetNet, mobile networks of the next generation can be proposed to benefit from extreme infrastructure densification, large quantities of new bandwidth and many more antennas on a very large scale. These combinations bring an opportunity to support a plethora of high-speed services for the bandwidthhungry applications [4].

The arrangement of three characteristics: ultra-dense networks (UDNs), mmWave and massive MIMO, which are fortunately symbiotic relationship is shown in Fig.8. The system is a HetNet made up of macrocell and small cell $B S s$, all with massive MIMO and mmWave communication capacities.

The wide bandwidth available and high spectrum efficiency make mmWave-massive MIMO a promising option to improve significantly the overall system throughput for future 5G cellular networks. The disadvantage of mmWave communications is the high path loss during the transmission. Usually mmWave communication systems use directional beamforming and combining by using large antenna arrays to reduce the path loss. A wide varity of challenges ranging the length and breadth of communications theory and engineering has to be addressed in order to harness the opportunities of mmWave-massive MIMO technology. Some of the areas of the challenges include [4]: information theoretic issues, channel modeling, channel estimation techniques, antenna and RF transceiver architecture design, waveforms and multiple access schemes, interference management, backhaul transmissions, mobility management, system-level modeling, standardization, etc. 


\section{5 mmWave-Massive MIMO Beamforming}

By regulating the phases and/or amplitudes of original signals, beamforming can optimize the performance of cellular networks using interference cancelation concept. It is a technique of preprocessing signals prior to transmission so as to maximize the network throughput and reliability by exploiting the available spatial degrees of freedom in a MIMO system [40]. There are primarily three categories of beam-forming architectures which are commonly explored: the analog or radio frequency beamforming, the digital beamforming and the hybrid beamforming architecture [41].

\subsection{Analog Beamforming}

Analog beamforming is the method of beamforming in which the control of MIMO and beamforming is done at $\mathrm{RF}$ level. In this case, a transceiver is believed to drive the antenna array. The transmit and receive array processing is performed with $\mathrm{RF}$ components having phase shifting and potentially gain adjustment capabilities [42]. In such a system, only a single RF chain with many analog phase shifters is employed to transmit a single data stream as shown in Figure 9. a). Analog beamforming is utilized to control the phases of original signals to achieve the maximal antenna array gain and effective SNR.

Analog beamforming has a basic hardware structure which is easier to implement. However, this beamforming has low antenna gain and suffers from serious performance loss, since only the phases of the transmit signals can be controlled but not their amplitudes. Thus, it is not practically used in Massive MIMO and mmWave communication systems [3].

\subsection{Digital Precoding}

It is a traditional technology commonly used in lowfrequency MIMO systems. The conventional MIMO systems comprise only a small number of antenna elements, typically less than ten. To provide higher degrees of freedom for the precoding in the traditional MIMO, each antenna element is equipped with a dedicated RF chain.

The precoding scheme in such a system can exploit this architecture by employing the precoders in the digital baseband, which permits tuning of both magnitude and phase values of transmit signals of each antenna element independently. Such a transceiver architecture, with a dedicated RF chain for each antenna element, is referred to as fully-digital precoding architecture [43] as shown in Figure 9. b). The basic principle of digital precoding is to monitor both phases and amplitudes of original signals in order to eliminate interferences in advance. It requires a dedicated baseband and RF chain for each antenna element. In smallscale MIMO precoding schemes such as zero forcing
(ZF) precoding, the symbols are modified in both amplitudes and phase at the baseband and supported by a dedicated radio frequency (RF) transceivers. Therefore, each antenna element requires a dedicated $\mathrm{RF}$ transceivers for assistance which is too expensive in the case of mmWave-massive MIMO.

\subsection{Hybrid Analog-digital Precoding}

Hybrid precoding offers a compromise between system performance and hardware complexity. One solution to keep the energy consumption, implementation cost and complexity of massive MIMO at affordable levels is to perform analog beamforming, operating in the $\mathrm{RF}$ domain, which requires a much smaller number of baseband ADCs, instead of power-hungry digital signal processing. Unfortunately, complete analog beamforming still has some critical shortcomings, since it lacks flexibility and adaptability, sacrifices the overall system performance and entails reliability issues in hardware design, especially for mmWave signals. On the other hand, digital precoding achieves satisfying performance since it controls both the phases and amplitudes of signals. Unfortunately, due to a dedicated RF chain is required for each antenna, digital precoding has high energy consumption and hardware cost, which makes it difficult to be directly extended to mmWave-massive MIMO systems with a large number of antennas [44]. A realistic option is to conduct a portion of the processing with a reduced number of RF chains in the baseband (digital) and the remaining part in the RF band (analog). Such topologies can offer an optimal balance between analog and digital processing, reduce the complexity, power consumption and cost as the number of RF chains are much less than the number of antennas. In [42], it is declared that hybrid beamforming architectures combines the advantages of both analog and digital beamforming. That is why, a hybrid beam-forming architecture with analog phase shifters has emerged as an attractive opportunity mmWave-massive MIMO systems.

The antenna elements are grouped into analog subarrays within the massive hybrid array architecture. Only a phase shifter is assigned to a single antenna element while the other components are shared by all antenna elements in each sub-array. Every subarray is fed with only one digital input (in the transmitter). Hence, it outputs only one digital signal (at the receiver). All digital signals from all the sub-arrays are jointly processed in a digital processor (Figure 9. c)). Accordingly, hybrid beamforming is viable for mmWave-massive MIMO, with less RF chain than the number of antennas. Compared to the digital beamformer this shows only a negligible performance loss. The comparison between analog, digital and hybride precoders is shown in Table $1[42,43,45]$. 


\begin{tabular}{|c|c|c|c|}
\hline \multirow{2}{*}{ Features } & \multicolumn{3}{|c|}{ Beamforming Types } \\
\cline { 2 - 4 } & Analog Beamforming & Digital Precoding & Hybride Precoding \\
\hline Number of Streams & Single stream & Multi-stream & Multi-stream \\
\hline Number of Users & Single-user & Multi-user & Multi-user \\
\hline Signal Control Cabability & Phase Control only & Phase and amplitude control & Intermediate \\
\hline Degree of freedom & Least & Highest & Intermediate \\
\hline Implementation & Phase Shifters & ADC/DAC, mixer & Intermediate \\
\hline Hardware Requirement & Least & Highest & Intermediate \\
\hline Energy Consumption & Least & Highest & Intermediate \\
\hline Cost & Least & Highest & Near-Optimal \\
\hline Complexity & Least & Highest & is realistic \\
\hline Performance & Least & Optimal & Impractical \\
\hline $\begin{array}{c}\text { Suitability for mmWave } \\
\text { Massive MIMO }\end{array}$ & Unsuitable & & \\
\hline
\end{tabular}

Table 1: Comparison of Precoding Techniques

\subsection{Channel and System Model}

\section{A.Channel Model for mmWave-Massive MIMO}

The system is composed of single $B S$ with Massive MIMO antenna arrays (transmitter), mmWave wireless channel and User terminals (receivers) as shwon in shown in Fig.6.

The high free space path loss is a feature of mmWave transmission, leading to reduced spatial selectivity or scattering. Contrarily, the large tightly packed antenna arrays are mmWave transceiver characteristics, leading to high rates of antenna correlation. For this reason, a narrow band clustered channel representation is adopted based on the extended Saleh Valenzuela model. This model allows to capture characteristics in mmWave channels accurately. Using the clustered channel model, the channel matrix $H$ is assumed to be a sum of the contributions of $L$ propagation paths. Therefore, an adoption of a geometric channel model with $L_{k}$ scatters is used for the channel of the $k^{\text {th }}$ user. Thus, the $k^{\text {th }}$ user channel matrix $H_{k} \in \mathbb{C}^{N_{r} \times N_{t}}$ can be expressed as

$$
\mathbf{H}_{k}=\sqrt{\frac{N_{t} N_{r}}{L_{k}}} \sum_{l=1}^{L_{k}} \alpha_{k, l} \mathbf{a}_{k, r}\left(\theta_{k, l}\right) \mathbf{a}_{k, t}^{H}\left(\phi_{k, l}\right)
$$

where $N_{t}$ is the number of antennas at the $B S, N_{r}$ is the number of antennas at the $k^{t h}$ MS. $\alpha_{k, l}$ is the complex gain of the $l^{\text {th }}$ path, which includes the path loss with $\mathbb{E}\left|\alpha_{k, l}\right|^{2}=\beta, \beta$ is a normalization constant. The variables $\phi_{l} \in[0,2 \pi]$ and $\theta_{l} \in[0,2 \pi]$ are the $l^{\text {th }}$ path's azimuth angles of departure or arrival (AoDs/AoAs) of the transmitter and receiver respectively. $\mathbf{a}_{k, t}\left(\phi_{k, l}\right)$ is the $B S$ antenna array response vector, $\mathbf{a}_{k, r}\left(\theta_{k, l}\right)$ is the antenna array response vector of the $k^{t h} M S, L_{k}$ is the number of propagation paths for the channel of the $k^{t h} M S$ with $l \in\left[1,2, \ldots, L_{k}\right]$. The base station and every mobile station is assumed to get knowledge about the structure of their antenna arrays.
Thus, the array response vector for ULA is expressed as [34]

$$
a_{U L A}(\phi)=\frac{1}{\sqrt{N}}\left[1, e^{j \frac{2 \pi}{\lambda} d \sin (\phi)}, \ldots e^{j(N-1) \frac{2 \pi}{\lambda} d \sin (\phi)}\right]^{T}
$$

For UPAs, the array response vector is

$$
\begin{array}{r}
a_{U P A}(\phi, \theta)= \\
\frac{1}{\sqrt{N}}\left[1, e^{j \frac{2 \pi}{\lambda} d(x \sin (\phi) \sin (\theta)+y \cos (\theta))}, \ldots\right. \\
\left.e^{j \frac{\pi}{\lambda} d\left(\left(W_{1}-1\right) \sin (\phi) \sin (\theta)+\left(W_{2}-1\right) \cos (\theta)\right)}\right]^{T}
\end{array}
$$

where $\lambda$ denotes the wavelength, $d$ is the spacing between two antennas (typically, $d=\frac{\lambda}{2}$ ), $\phi$ is the azimuth angle, $\theta$ is the elevation (or zenith) angle and $N$ is the number of antenna elements.

B. Precoding Model for mm Wave-Massive MIMO

Consider the single-user mmWave-massive MIMO system with hybrid precoding as shown the same Figure in mmWave Communications (Fig. 6), where the BS employs $N$ antennas to simultaneously transmit $N_{s}$ data streams to a user with $M$ antennas.

To enable multistream transmission, the $B S$ is equipped with $N_{R F} R F$ chains such that $N_{s} \leq N_{R F} \leq$ $N$. The $B S$ applies an $N_{R F} \times N_{s}$ digital precoder $\mathbf{D}_{B B}$ using its $N_{R F} R F$ chains, followed by an $N \times N_{R F} R F$ analog beam former $\mathbf{A}_{R F}$ using analog circuitry such as phase shifters. Then, the transmitted signal can be presented by [4]

$$
\mathbf{x}=\mathbf{A}_{R F} \mathbf{D}_{B B} \mathbf{s}
$$

where $\mathbf{s}$ is $N_{s} \times 1$ original signal vector before precoding with normalized power as $\mathbb{E}\left(\mathbf{s s}^{H}\right)=\left(\frac{1}{N_{s}}\right) \mathbf{I} N_{s}$. For 
simplicity, we consider a narrow band system, which can be regarded as a reasonable first step because in mmWave frequnecy, coherence bandwidth is usually sufficiently broad. The received signal vector $\mathbf{y}$ of size $M \times 1$ can be correspondingly presented as

$$
\mathbf{y}=\sqrt{\rho} \mathbf{H} \mathbf{A}_{R F} \mathbf{D}_{B B} \mathbf{s}+\mathbf{n}
$$

We assume the channel matrix $\mathbf{H}$ is perfectly known at both the $B S$ and user to enable precoding.

For a multi-user hybrid precoding scheme, consider a mmWave-massive MIMO system with $B S$ antennas $N$ and $N_{R F}$ RF chains such that $N_{R F} \leq N$ and $K$ terminals each with $M$ antennas and only one $\mathrm{RF}$ chain. For a fully connected hybrid precoder system, the BS employs $K \times K$ digital precoder in the baseband $D_{k}=\left[D_{1}, D_{2}, \ldots D_{k}\right]$ followed by $N \times K$ analog precoder $A_{k}=\left[A_{1}, A_{2}, \ldots, A_{K}\right]$. The received signal vector $r_{k}$ observed by the $k^{t h}$ terminal after precoding can then be expressed as

$$
r_{k}=\mathbf{H}_{k} \sum_{n=1}^{K} \mathbf{A}_{n} \mathbf{D}_{n} \mathbf{s}_{n}+\mathbf{n}_{k}
$$

After being combined with the analog combiner $w_{k}$, where $w_{k}$ has similar constraints as the analog precoder $A_{k}$, the signal $y_{k}$ becomes

$$
y_{k}=\mathbf{w}_{K}^{H} \mathbf{r}_{k}=\mathbf{w}_{K}^{H} \mathbf{H}_{k} \sum_{n=1}^{K} \mathbf{A}_{n} \mathbf{D}_{n} \mathbf{s}_{n}+\mathbf{w}_{K}^{H} \mathbf{n}_{k}
$$

The goal of aim of hybrid precoding is to design the analog precoder $A$, the digital precoder $D$ at the basestation and the analog combiners $\left\{w_{k}\right\}_{k}^{K}=1$ at the $M S$ to boost the sum rate $R$.

Then, the received signal-to-interference-plus-noiseratio (SINR) for the $k^{t h}$ user can be obtained as

$$
\gamma_{k}=\left(\frac{\frac{P}{K}\left|\mathbf{w}_{k}^{H} \mathbf{H}_{k} \mathbf{A} \mathbf{D}_{u}\right|^{2}}{\frac{P}{K} \sum_{n \neq k}^{\max }\left|\mathbf{w}_{k}^{H} \mathbf{H}_{k} \mathbf{A} \mathbf{D}_{u}\right|^{2}+\sigma_{n}^{2}}\right)
$$

Then, the system capacity is

$$
R=B \sum_{k=1}^{K} \log _{2}\left(1+\gamma_{k}\right)
$$

where, $B$ is the system bandwidth.

\section{C.Power Model for mmWave-Massive MIMO}

Typically, the power consumption for a system comprises radiated power, circuit power and power consumed for signal processing. In our case, the total power consumption is usually modeled as [46]

$$
P_{T}=P_{t}+N_{R F} P_{R F}+N_{S} P_{P S}
$$

where $P_{t}$ is the transmission power, $P_{R F}$ is the power consumed by $R F$ chain and $P_{S}$ is the energy consumption of power supply, $N_{R F}$ and $N_{P S}$ are the numbers of $\mathrm{RF}$ chains and power supplies respectively.

\section{Spectral and Energy Efficiency Model for mm Wave-} Massive MIMO

The energy and spectral efficiencies are two tradeoff parameters. Energy efficiency metric is a "green communication" indicator. It is a function of the data rate over the mmWave links. Fundamentally, the significant improvement of energy efficiency is achieved by extremely sharpening the radiated energy from the transmitter to focus into small regions where active user equipment located. The energy efficiency is examined as a function of $B S$ serving distance, the transmission power and the SNR [47]. The energy efficiency degrades as the transmission power increases. It is the ratio of the achievable rate to the total power consumption.

Because spectral efficiency is directly proportional to the transmit power, the signal power must be increased to improve the system's spectral efficiency. On the other hand, as the signal power increases, the power usage in the system increases and the energy efficiency in turn reduces.By using moderately large antenna arrays, however, we can jointly enhance the spectral and energy efficiency compared with single antenna system in orders of magnitude. The $S E$ and $E E$ of the downlink cellular network can be expressed as [13]

$$
\eta_{S E}=\frac{R}{B}, \quad \eta_{E E}=\frac{B \eta_{S E}}{P_{T}}
$$

\section{Results and Discussion}

In this section, the performance of hybrid precoding for mmWave-massive MIMO is evaluated in terms of SNR, data rate, achievable rate and energy efficiency.

It can be seen from Figure 10 that as the SNR increases, the spectral efficiency of the different precoding techniques is improved to different degrees. Similarly, for all data streams, the performance of the fully 
digital precoding is the best because it is the optimal precoding method and all the other precoding techniques are aimed at approaching it. The performance of hybrid precoding is close to the full-digital algorithm and better than the traditional analog precoding. The analog precoding performance is the worst of all precoding schemes.

Fig.11 shows that even at very large numbers of antennas, there is still a considerable gain of hybrid precoding over analog beam steering. We can also observe from this plot that the difference between the spectral efficiency achieved by the hybrid precoding and digital precoding decreases when the number of BS antennas increases. This can be explained by the fact that the performance improvement obtained by the digital precoder becomes obvious with the increasing number of $\mathrm{RF}$ chains, and the fully analog precoder system will suffer from a non-negligible performance loss.

The spectral efficiency with SNR achieved by different linear precoding methods is shown in Fig.12. It is observed that hybrid linear precoding schemes outperforms the analog one in the whole SNR range, and the performance gap becomes more obvious with the increasing SNR. For example, when SNR $=-15 \mathrm{~dB}$, the capacity gap between the the linear precoders and the single user is less than $1 \mathrm{bps} / \mathrm{Hz}$, while when $\mathrm{SNR}=10$ $\mathrm{dB}$, the capacity gap increases up to $5 \mathrm{bps} / \mathrm{Hz}$. The analog precoding performance is the worst because the analog precoding is constant modulus; only the phase characteristics are utilized, and the amplitude characteristics are not utilized.

Fig. 13 compares the averaged achievable rates by varying the number of users for a fixed $\mathrm{SNR}=10$ $\mathrm{dB}$ in a multi-path scenario based on hybrid linear precoding schemes. We found that the hybrid precoder MRT decrease its performance for higher number of users, whereas, the hybrid precoders ZF and MMSE scheme show good outcomes.

The result in Figure 14 shows the energy efficiency with the number of $B S$ antennas. As shown in the plot, as the number of BS antenna increases, the power consumption also increases, hence the energy efficiency decreases. This is because the overall circuit power consumption of the BS increases as the number of BS antennas grows high. This results in a lower energy efficiency. Thus, even if it may help to reduce the transmitting power for the network by raising the number of BS antennas, it also reduces the energy efficiency because of the raise in internal power consumption. As a result, the energy efficiency of digital precoding becomes the worst. On the other hand, hybrid bemaforming has better enery efficiency than its counterpart digital precoding.

\section{Conclusion}

This work covers a comprehensive overview that focuses on the promising technologies that are needed in $5 \mathrm{G}$ and beyond mobile systems. A complete overview of the massive MIMO and its advantages on $5 \mathrm{G}$ technology is mentioned and preceded by analysis on mmWave communication that is recognized as a promising technology that will play a major role in $5 \mathrm{G}$ network systems. Subsequently, we presented a brief details on channel estimation, signal processing and beamforming techniques. In doing so, we highlighted the peculiarities of the emerging network technologies of massive MIMO, mmWave communication and mmWave-massive MIMO systems. It also examines the type of precoding techniques being proposed for each technology. Thus, the comparison and performance of analog, digital and hybrid analog-digital precoders under perfect CSI at the transmitters is addressed. Hybrid precoding, which combines the advantages of both digital and analog beamforming is analyzed. Hybrid precoding, with only a small number of RF chains, can achieve performance quite close to digital precoding. Consequently, hybrid precoding seems more suitable for future mmWave-massive MIMO systems. The simulation results also show that hybrid linear precoding schemes achieves a higher spectral efficiency compared with analog and digital which confirms the better management of the multi-user interference by hybrid analog-digital scheme. Finally, the hybrid linear precoding schemes achieves high data rates due to a large amount of spatial degrees of freedom available in mmWave massive MIMO systems absolutely makes it one of the most powerful technology that enhances the performance of $5 \mathrm{G}$ and beyond networks.

\section{Future Research Directions}

There are still many issues for mmWave-massive MIMO that are open for discussion and necessitate finalization. The concepts and techniques which are under research are energy efficiency, spectral efficiency, analytical modeling, channel and field measurement campaigns, test and validation of system performance, are still ongoing with different researchers. In addition, various algorithms, schemes and techniques are being proposed for mmWave-massive MIMO systems in the areas of modulation and signal waveform, multiple access scheme, front haul design, antenna array architectur and precoding schemes are some of them. Thus, the integrated performance characterization and evaluation in real-life scenarios and applications of such mmWave-massive MIMO techniques remains an open question. 


\section{List of Abbreviations}

5G: Fifth Generation; AOA: Angle of Arrival; AoD: Angle of Departure; BS: Basestation; CSI: Channel State Information; DoF: Degrees of Freedom; EE: Energy Efficiency; iid: independent and identically distributed; LoS: Line of Sight; MAC: Media Access Control; MF: Matched Filter; MIMO: Multiple Input Multiple Output; MMSE: Minimum Mean Square Error; MRT: Maximum Ratio Transmission; mmWave: Millimeter Wave; MS: Mobile Station; NLoS: Non Line of Sight; PZF: Phased Zero Forcing; RF: Radio Frequency; RZF: Regularized Zero Forcing; SE: Spectral Efficiency; SINR: signal to interference noise ratio; SNR: Signal to Noise Ratio; TDD: Time Division Duplex; TPE: Truncated Polynomial Expansion; UDNs: Ultra Dense Networks; ULA: Uniform Linear Array; UPA: Uniform Planner Array; ZF: Zero Forcing;

\section{Competing Interests}

The authors declare that they have no competing interests.

\section{Author's Contributions}

All authors read and approved the final manuscript.

\section{Acknowledgment}

The authors would like to thank the anonymous reviewers for their valuable comments and suggestions that helped improve the quality of this manuscript.

\section{Funding}

Not applicable.

\section{Availability of Data and Materials}

Not available online. Please contact the author for data requests.

\section{Authors' Information}

Tewelgn Kebede Engda is a PhD Student. He is conducting his PhD research. Yihenew Wondie (Dr.Eng.) is the head of the Postgraduate programs and Lecturer. Johannes Steinbrun (Prof.Dr.Ing. Dr.h.c.mult.) is an emeritus professor. Both are Supervisors of Tewelgn Kebede Engda.

\section{Author details}

${ }^{1}$ Addis Ababa Institute of Technology, Addis Ababa University, Addis Ababa, Ethiopia. ${ }^{2}$ Addis Ababa Institute of Technology, Addis Ababa University, Addis Ababa, Ethiopia. ${ }^{3}$ Faculty of Electrical Engineering, Kempten University of Applied Sciences, Kempten, Germany.

\section{References}

1. Vincent W. S. Wong, Robert Schober, Derrick Wing Kwan Ng and Li-Chun Wang, "Key Technologies for 5G Wireless Systems," Cambridge University Press 2017, UK, 2017.

2. Yang Yang, Jing Xu, Guang Shi and Cheng-Xiang Wang, "5G Wireless Systems Simulation and Evaluation Techniques," (C) Springer International Publishing AG, 2018.

3. Shahid Mumtaz, Jonathan Rodriguez and Linglong Dai, "mmWave Massive MIMO: A Paradigm for 5G," (C) Elsevier Inc. All, 2017.

4. Sherif Adeshina Busari, Kazi Mohammed Saidul Huq, Shahid Mumtaz, Linglong Dai and Jonathan Rodriguez, "Millimeter-Wave Massive MIMO Communication for Future Wireless Systems: A Survey," IEEE Communications Surveys and Tutorials, Vol. 20, NO. 2, 2nd Quarter,2018.

5. Qian Li, Huaning Niu, Apostolos Papathanassiou and Geng Wu, "5G network capacity: Key elements and technologies," IEEE Veh. Technol. Mag., vol. 9, no. 1, pp. 71-78, Mar. 2014.

6. T. E. Bogale and L. B. Le, "Massive MIMO and mmWave for $5 \mathrm{G}$ wireless HetNet: Potential benefits and challenges," IEEE Veh. Technol. Mag., vol. 11, no. 1, pp. 64-75, Mar. 2016.

7. Mohamed Alouzi and Francois Chan, "Performance of Millimeter Wave Massive MIMO with the Alamouti Code," 978-1-5386-6355-4/18/IEEE, 2018.

8. Wei Wang and Wei Zhang, "Antenna Subset Selection for Line-of-Sight Millimeter Wave Massive MIMO Systems," 978-1-74052-390-5/17/IEEE, 2017.

9. Mamta Agiwal, Abhishek Roy and Navrati Saxena, "Next Generation 5G Wireless Networks: A Comprehensive Survey," IEEE Communications Surveys and Tutorials, Vol.18, NO.3, 2016.

10. Rath Vannithamby and shilpa Talwar, "Towards 5G Aapplications, Requirements and Candidate Technologies," John Wiley and Sons, Ltd, USA, 2017.
11. Mahmoud A. Albreem, Markku Juntti and Shahriar Shahabuddin, "Massive MIMO Detection Techniques: A Survey," IEEE Communications Surveys and Tutorials, Vol. 21, NO. 4, Fourth Quarter, 2019.

12. E. Björnson, E. G. Larsson, and M. Debbah, "Massive MIMO for maximal spectral efficiency: How many users and pilots should be allocated?," IEEE Trans. Wireless Commun., vol. 15, no. 2, pp. 1293-1308, Feb. 2016.

13. J. Chen, H. Chen, H. Zhang, and F. Zhao, "Spectral-energy efficiency tradeoff in relay-aided massive MIMO cellular networks with pilot contamination," IEEE Access, vol. 4, pp. 5234-5242, 2016.

14. E. Björnson, E. G. Larsson, and T. L. Marzetta, "Massive MIMO: Ten myths and one critical question", IEEE Commun. Mag., vol. 54, no. 2, pp. 114-123, Feb. 2016.

15. Erik G. Larsson and Liesbet Van der Perre, "Massive MIMO for 5G," IEEE 5G Tech Focus: Volume 1, Number 1, March 2017.

16. T. T. Do, E. Björnson, E. G. Larsson, and S. M. Razavizadeh, "Jamming-resistant receivers for the massive MIMO uplink," IEEE Trans. Inf. Forensics Security, vol. 13, no. 1, pp. 210-223 2018.

17. E. G. Larsson, O. Edfors, F. Tufvesson, and T. L. Marzetta, "Massive MIMO for next generation wireless systems," IEEE Commun. Mag., vol. 52, no. 2, pp. 186-195, Feb. 2014.

18. Furqan Jameel, Faisal, M. Asif Ali Haider and Amir Aziz Butt, "Massive MIMO: A Survey of Recent Advances, Research Issues and Future Directions," 978-1-5386-2234-6/17/ IEEE, 2017.

19. X. Gao, O. Edfors, F. Rusek, and F. Tufvesson, "Linear pre-coding performance in measured very-large mimo channels," IEEE Vehicular Technology Conference, Nov. 2011.

20. Olakunle Elijah, Chee Yen Leow, Tharek Abdul Rahman, Solomon Nunoo and Solomon Zakwoi Iliya, "A Comprehensive Survey of Pilot Contamination in Massive MIMO-5G System," IEEE Communications Surveys and Tutorials, Vol. 18, NO. 2, , SECOND QUARTER 2016.

21. Erik G. Larsson, Ove Edfors, Fredrik Tufvesson, Thomas L. Marzetta, "Massive MIMO for Next Generation Wireless Systems," IEEE Communications Magazine, February 2014.

22. A. Zaib, . Masood, A. Ali, W. Xu, and T. Y. Al-Naffouri, "Distributed channel estimation and pilot contamination analysis for massive MIMO-OFDM systems," IEEE Trans. Commun., vol. 64, no. 11, pp. 4607-4621, Nov. 2016.

23. Varna L. Babu, Luxy Mathews, Sakuntala S. Pillai, "Performance Analysis of Linear and Nonlinear Precoding in MIMO Systems," International Journal of Advanced Research in Computer and Communication Engineering Vol. 4, Issue 6, June, 2015.

24. Lu Lu, Geoffrey Ye Li, A. Lee Swindlehurst, Alexei Ashikhmin and Rui Zhang, "An Overview of Massive MIMO: Benefits and Challenges," IEEE Journal of Selected Topics in Signal Processing, Vol. 8, NO. 5, October 2014.

25. Tewelgn Kebede, Amare Kassaw, Yihenew Wondie, Johannes Stenbrunn, "Joint Evaluation of Spectral Efficiency, Energy Efficiency and Transmission Reliability in Massive MIMO Systems," International Conference on Advances of Science and Technology ICAST 2019: Advances of Science and Technology pp 424-435, 2019.

26. Mohammad Mohammad, Abdalla Osman and Nihad Elhag, "Performance comparison of MRT and ZF for single cell downlink massive MIMO system," IEEE International Conference on Computing, Control, Networking, Electronics and Embeddeds Systems Engineering, 2015.

27. Bekele Mulu Zerihun and Yihenew Wondie, "Massive MIMO for $5 \mathrm{G}$ Cellular Networks: Potential Benefits and Challenges," ICST Institute for Computer Sciences, Social Informatics and Telecommunications Engineering, 2018.

28. Xiong Wang, Linghe Kong, Fanxin Kong, Fudong Qiu, Mingyu Xia, Shlomi Arnon, Member and Guihai Chen, "Millimeter Wave Communication: A Comprehensive Survey," IEEE Comminications Surveys and Tutorials, Vol. 20, NO. 3, 3rd Quarter ,2018.

29. Wei Xiang, Kan Zheng and Xuemin (Sherman) Shen, "5G Mobile Communications," Springer International Publishing Switzerland, 2017.

30. T. Rappaport, S. Sun, R. Mayzus, H. Zhao, Y, Azar, K, Wang, G. Wong, J. Schulz, M. Samimi, and F. Gutierrez, "Millimeter wave mobile communications for $5 \mathrm{G}$ cellular: It will work!," IEEE Access, vol. 1, pp. 335-349, May 2013. 
31. Ananya Das and S. Kolangiammal, "Performance Analysis of Millimeter Wave Communication System Using 256-QAM and 512-QAM Techniques," IEEE International Conference on Communication and, April 2017.

32. Ming Xiao et al, "Millimeter Wave Communications for Future Mobile Networks," IEEE IEEE ournal on Selected Areas in Communications, Vol. 35, NO. 9, September 2017.

33. A. Ghosh, T.A. Thomas, et al., "Millimeter-Wave Enhanced Local Area Systems: A High-Data-Rate Approach for Future Wireless Networks," IEEE Journal on Selected Areas in Communications, Vol. 32, NO. 6, JUNE 2014.

34. Dian-Wu Yue, Shuai $\mathrm{Xu}$ and Ha H. Nguyen, "Diversity gain of millimeter-wave massive MIMO systems with distributed antenna arrays," EURASIP Journal on Wireless Communications and Networking,, 2019.

35. Yahia R. Ramadan and Hlaing Minn, "Novel Hybrid Precoding Designs for mmWave Multiusern Systems with Partial Channel Knowledge," IEEE 978-1-5090-5019-2/17, 2017.

36. Ahmed Alkhateeb, Omar El Ayach, GeertLeus and Robert W. Heath, Jr., "Channel Estimation and Hybrid Precoding for Millimeter Wave Cellular Systems," IEEE Journal of Selected Topics in Signal Processing, Vol. 8, NO. 5,, Oct 2014.

37. Yu Huang, Chen Liu, Yunchao Song and Xiaolei Yu, "DFT codebook-based hybrid precoding for multiuser mmWave massive MIMO systems," EURASIP Journal on Advances in Signal Processing, https://doi.org/10.1186/s13634-020-00669-4, 2020.

38. A. L. Swindlehurst, E. Ayanoglu, P. Heydari, and F. Capolino, "Millimeter-wave massive MIMO: The next wireless revolution?," IEEE Commun. Mag., vol. 52, no. 9, pp. 56-62, Sep 2014.

39. Yuhan Sun and Chenhao Qi, "Weighted Sum-Rate Maximization for Analog Beamforming and Combining in Millimeter Wave Massive MIMO Communications," IEEE Ccommunictions Letters, Vol. 21, NO. 8 , August 2017.

40. Ganapati Hegde, "Energy-Efficient and Robust Hybrid Analog-Digital Precoding for Massive MIMO Systems," Darmstadt Technical University Dissertation (https://tuprints.ulb.tu-darmstadt.de/9210/), Germany 2019.

41. J. Brady and A. Sayeed, "Beamspace MU-MIMO for high-density gigabit small cell access at millimeter-wave frequencies," IEEE 15th International Workshop on Signal Processing Advances in Wireless Communications, 2014

42. Irfan Ahmed, Hedi Khammari, Adnan Shahid, Ahmed Musa, Kwang Soon Kim, Eli De Poorter, Ingrid Moerman, "A Survey on Hybrid Beamforming Techniques in 5G: Architecture and System Model Perspectives," Journal of Latex Class Files, Vol.XX, NO. X, August 2018.

43. X. Gao, L. Dai, S. Han, C. I, and R. W. Heath, "Energy-efficient hybrid analog and digital precoding for mmWave MIMO systems with large antenna arrays," IEEE EEE J. Select. Areas Commun., vol. 34, no. 4, pp.998-1009, April 2016.

44. Lu Lu, Geoffrey Ye Li, A. Lee Swindlehurst, Alexei Ashikhmin and Rui Zhang, "An Overview of Massive MIMO: Benefits and Challenges," IEEE Journal of Selected Topics in Signal Processing, Vol. 8, NO. 5, October 2014.

45. Baghdad Hadji, Lamya Fergaani and Mustapha Djeddou, "Hybrid Linear Precoding Strategy for Multiuser Massive MIMO Millimeter Wave Systems," IEEE 978-1-7281-3156-6/19, 2019.

46. YANG LIU, QINGXIA FENG, QIONG WU, YINGHUI ZHANG, MINGLU JIN, (Member, IEEE), AND TIANSHUANG QIU,

"Energy-Efficient Hybrid Precoding With Low Complexity for mmWave Massive MIMO System," IEEE Access, Digital Object Identifier 10.1109/ACCESS.2019.2928559, August 1, 2019

47. Abdulbaset M. Hamed and Raveendra K. Rao, "Spectral and Energy Efficiencies in mmWave Cellular Networks for Optimal Utilization," Hindawi Wireless Communications and Mobile Computing Volume 2018, Article ID 3097094, https://doi.org/10.1155/2018/3097094, 2018.

Figures
Figure 1: Amalgam of UDN, Massive MIMO and $\mathbf{m m W a v e}[4]$. A symbiotic relationship between UDN, Massive MIMO and mmWave.

Figure 2: Massive MIMO System Model [11]. This Figure shows the downlink Massive MIMO System Model [11].

Figure 3: Performance of Massive MIMO Linear Precoders. The performance comparison of Shanon Capacity with linear precoders called: ZF, MRT and RZF.

Figure 4: Spectral Efficiency Versus Signal to Noise Ratio. The Spectral efficiency of ZF, MRT and RZF is compared with their signal to noise ratio.

Figure 5: Atmospheric and Molecular Absorption at mmWave Frequencies [28]. Atmospheric and Molecular Absorption at mmWave Frequencies.

Figure 6: Hybrid Analog-digital Precoding in mmWave Communications [34]. System Model for Hybrid Analog-digital Precoding in mmWave Communications.

Figure 7: Antenna Array Structure [4]. The different array structure of Antennas.

Figure 8: Candidate Network Architecture with mmWave-Massive MIMO for 5G [3]. The arrangement of UDNs, mmWave and massive MIMO communications.

Figure 9: Types of Beamforming Architectures [42]. The different types of Beamforming Architectures. 
Figure 10: Achievable Rate Versus SNR. The spectral efficiency comparison of the different beamforming techniques for mmWave-Massive MIMO System.

Figure 11: Spectral Efficiency with $\boldsymbol{B} \boldsymbol{S}$ Antennas. The Performance comparison of the different beamforming techniques with resoect to the number of basestation antennas.

Figure 12: Spectral Efficiency Versus SNR with different Precoding Schemes. The Spectral efficiency of the different types of beamforming techniques are compared with respect to signal to noise ratio.

Figure 13: Achievable Rate Versus Number of Users. The Spectral efficiency of the different types of beamforming techniques are compared with respect to the number of users.

Figure 14: Energy Efficiency Versus Number of Base Station Antennas. The comparison of the different beamforming techniques with number of basesation antennas. The result shows their energy efficiency. 


\section{Figures}

\section{Ultra-Dense Network}
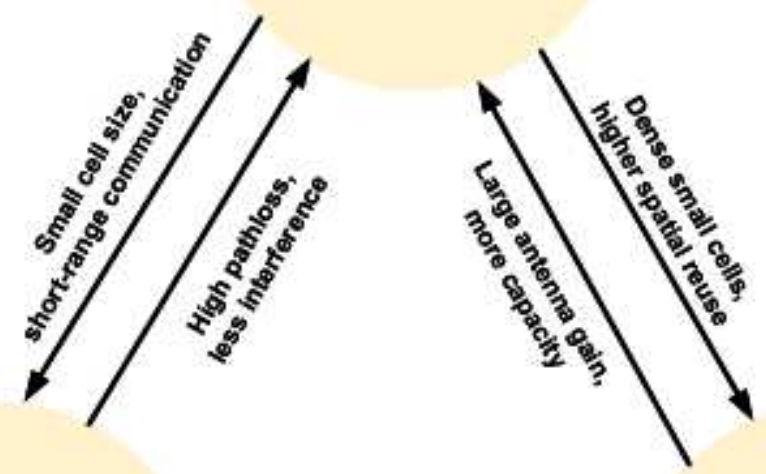

Short wavelength,

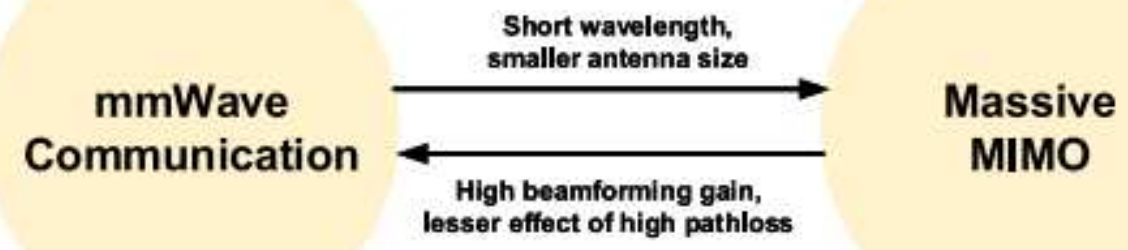

Figure 1

Amalgam of UDN, Massive MIMO and mmWave [4]. A symbiotic relationship be- tween UDN, Massive MIMO and mmWave. 


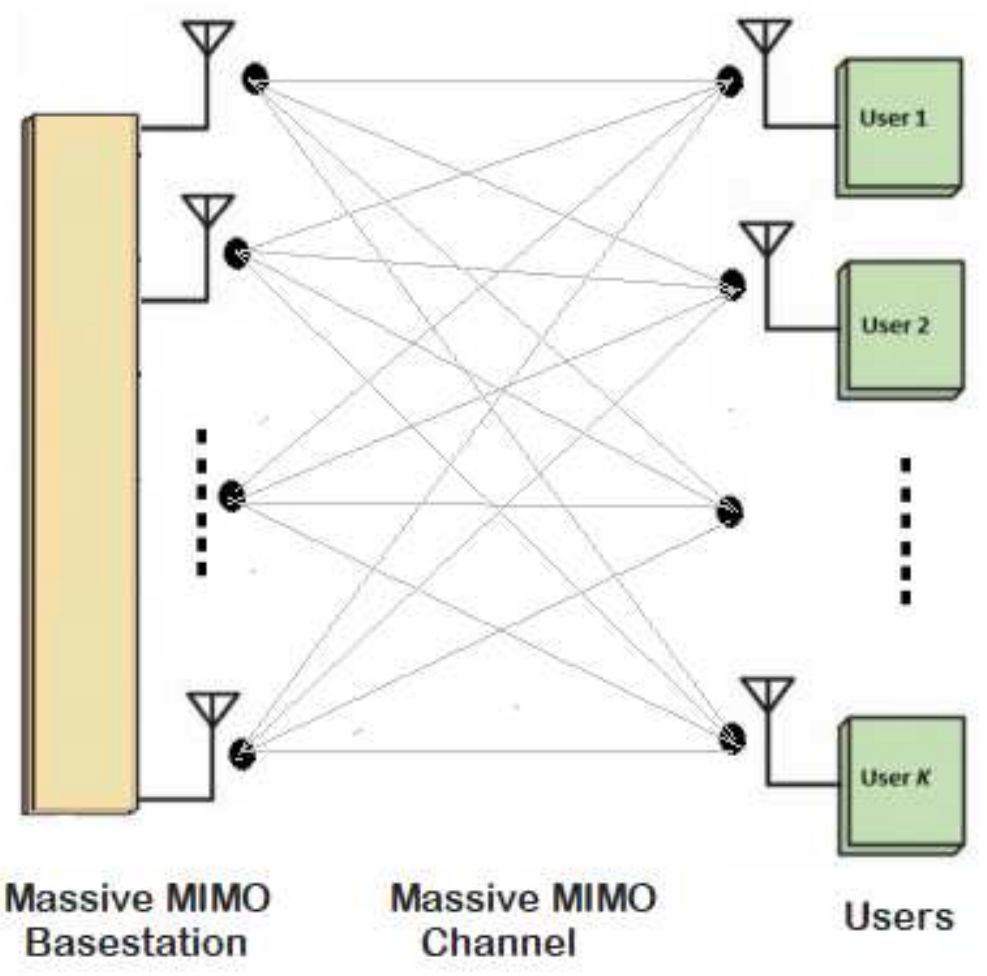

Figure 2

Massive MIMO System Model [11]. This Figure shows the downlink Massive MIMO Sys- tem Model [11].

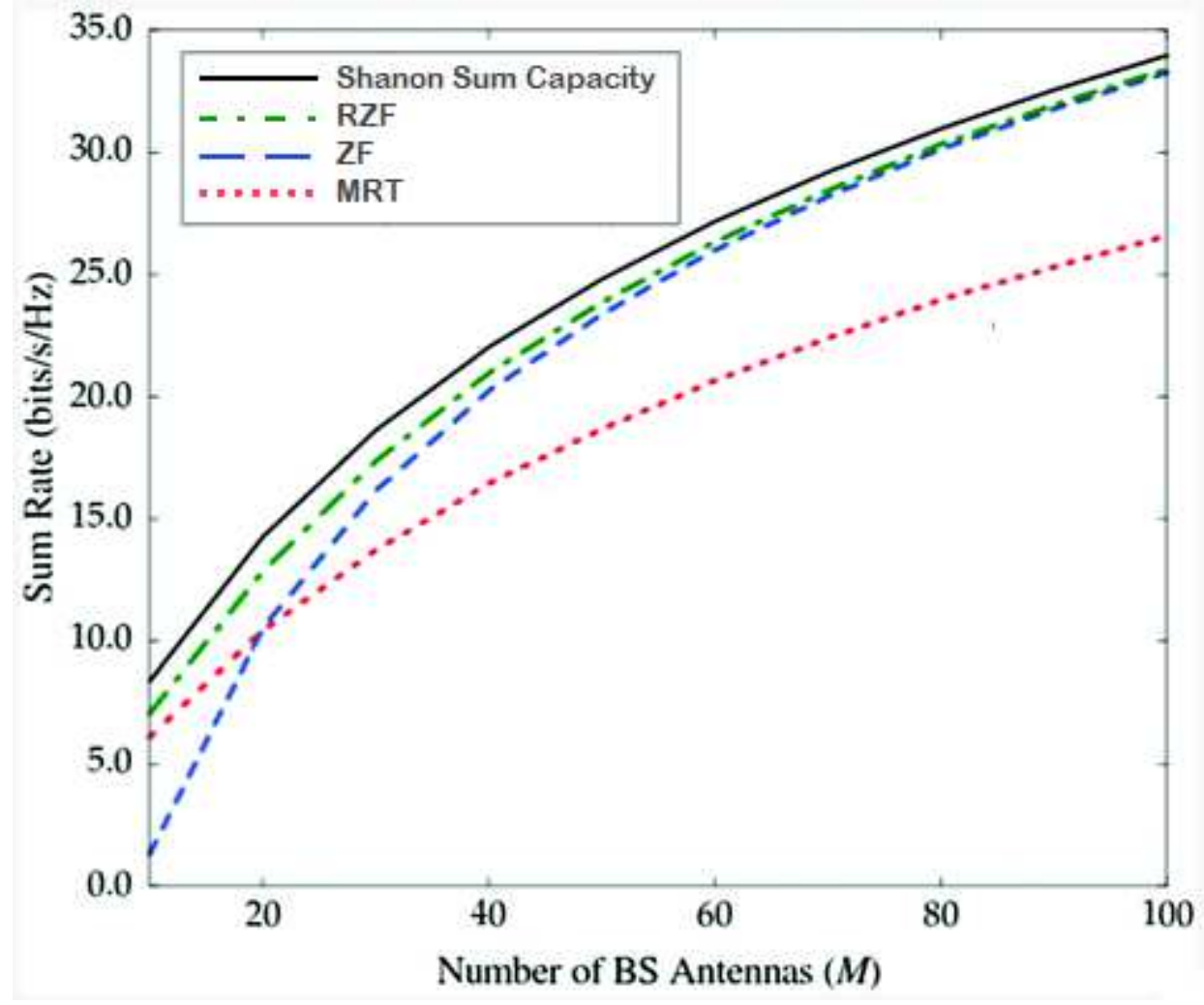

Figure 3 
Performance of Massive MIMO Lin- ear Precoders. The performance comparison of Shanon Capacity with linear precoders called: ZF, MRT and RZF.

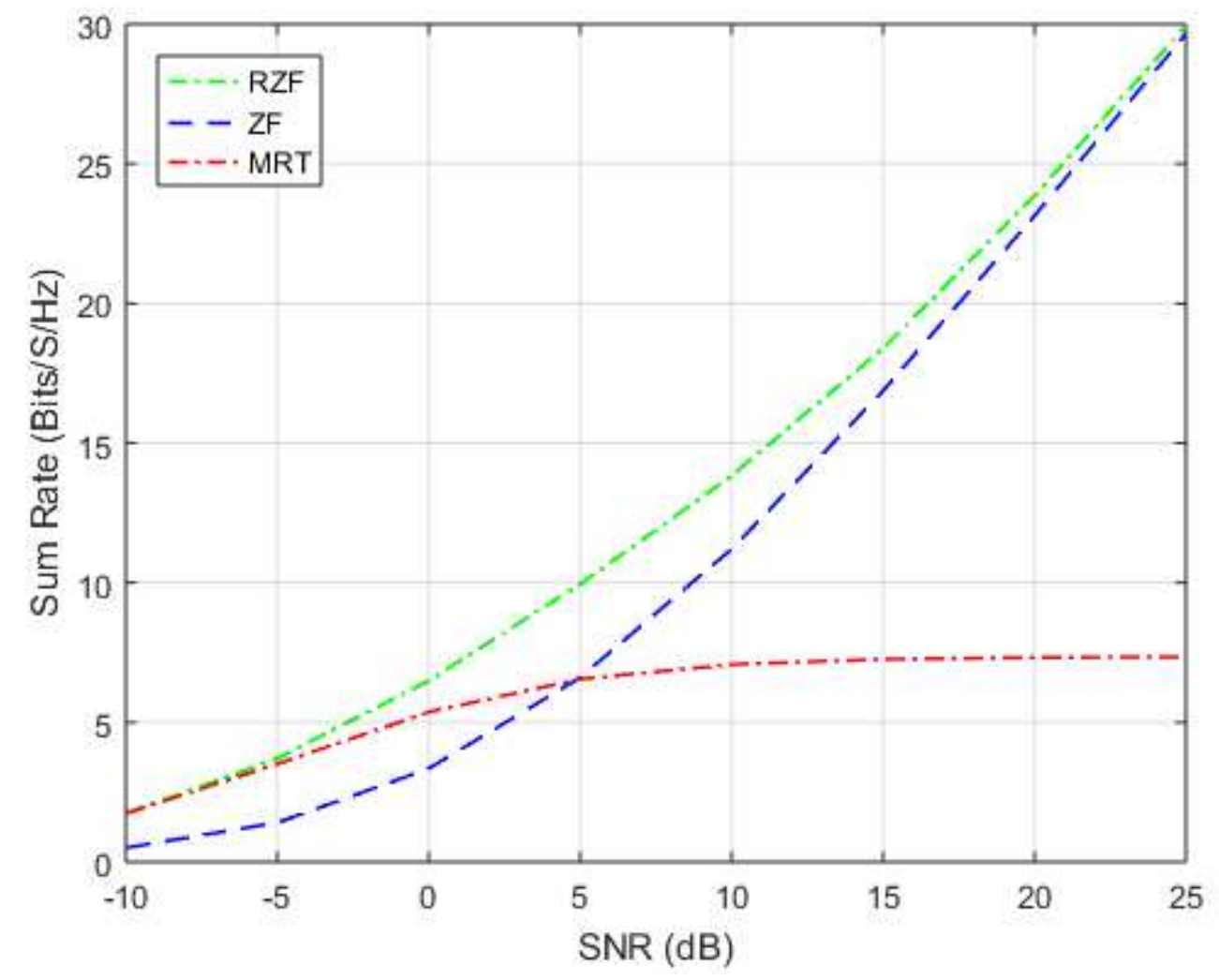

Figure 4

Spectral Efficiency Versus Signal to Noise Ratio. The Spectral efficiency of ZF, MRT and RZF is compared with their signal to noise ratio.

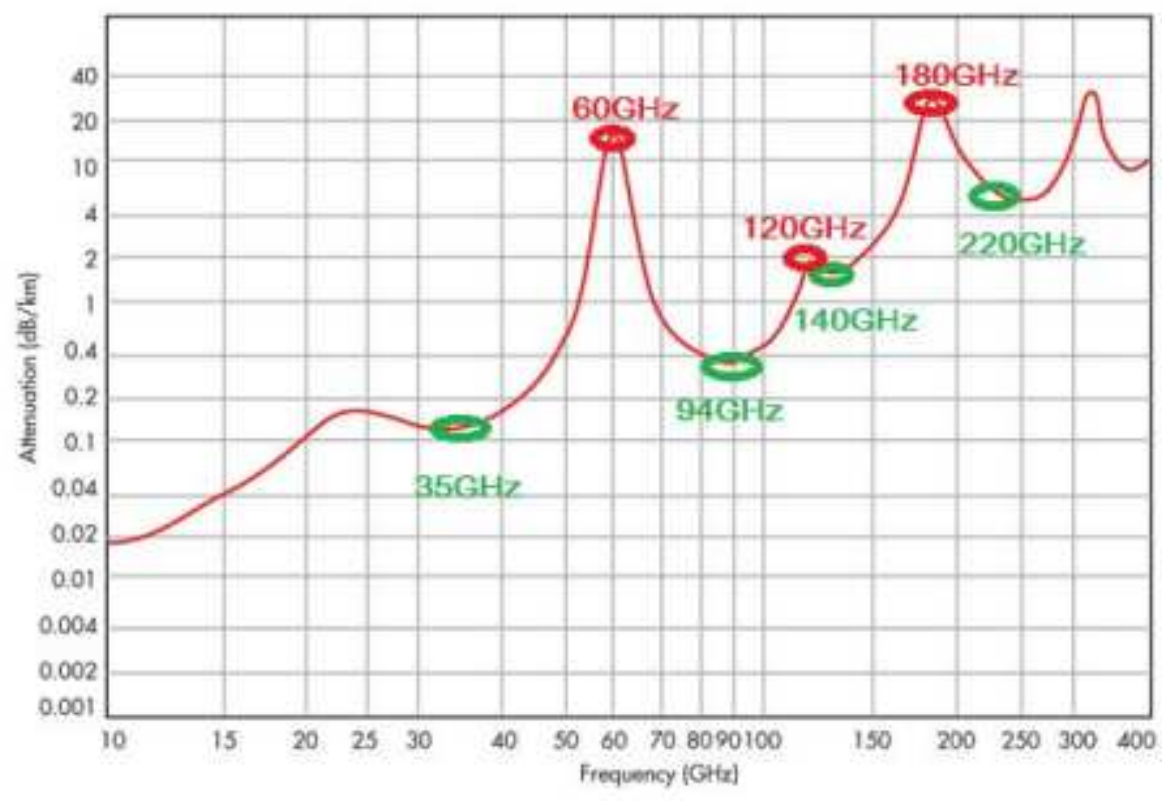

Figure 5 
Atmospheric and Molecular Absorption at mmWave Frequencies [28]. Atmospheric and Molecular Absorption at mmWave Frequencies.

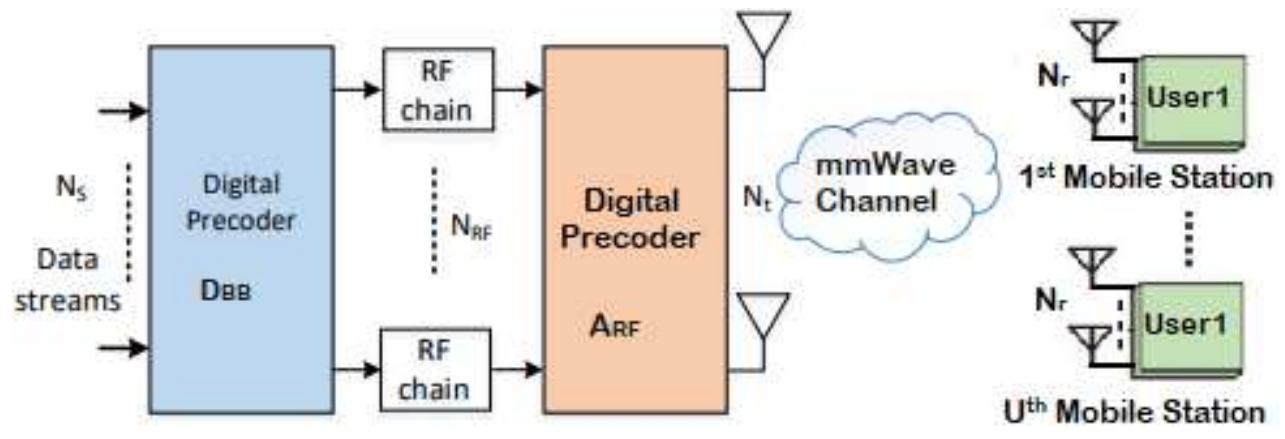

\section{Figure 6}

Hybrid Analog-digital Precoding in mmWave Communications [34]. System Model for Hybrid Analogdigital Precoding in mmWave Communications.

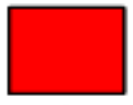

1

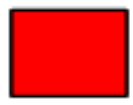

2

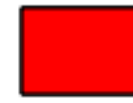

3

a) Uniform Linear Array
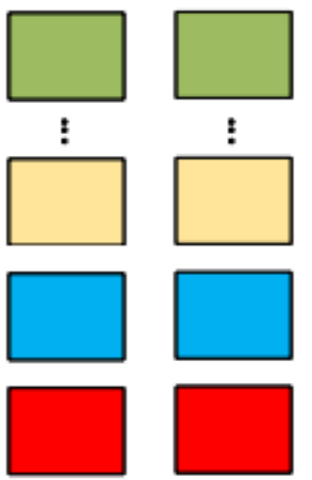

2

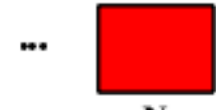

$\mathbf{N}$

b) Planar Linear Array

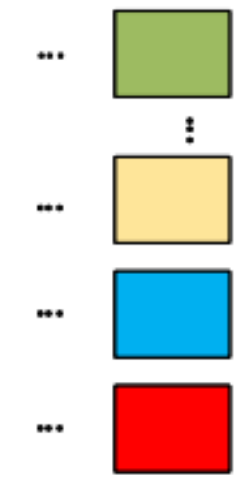

$\mathrm{W}_{1}$

\section{Figure 7}

Antenna Array Structure [4]. The different array structure of Antennas. 


\section{D2D Channel}

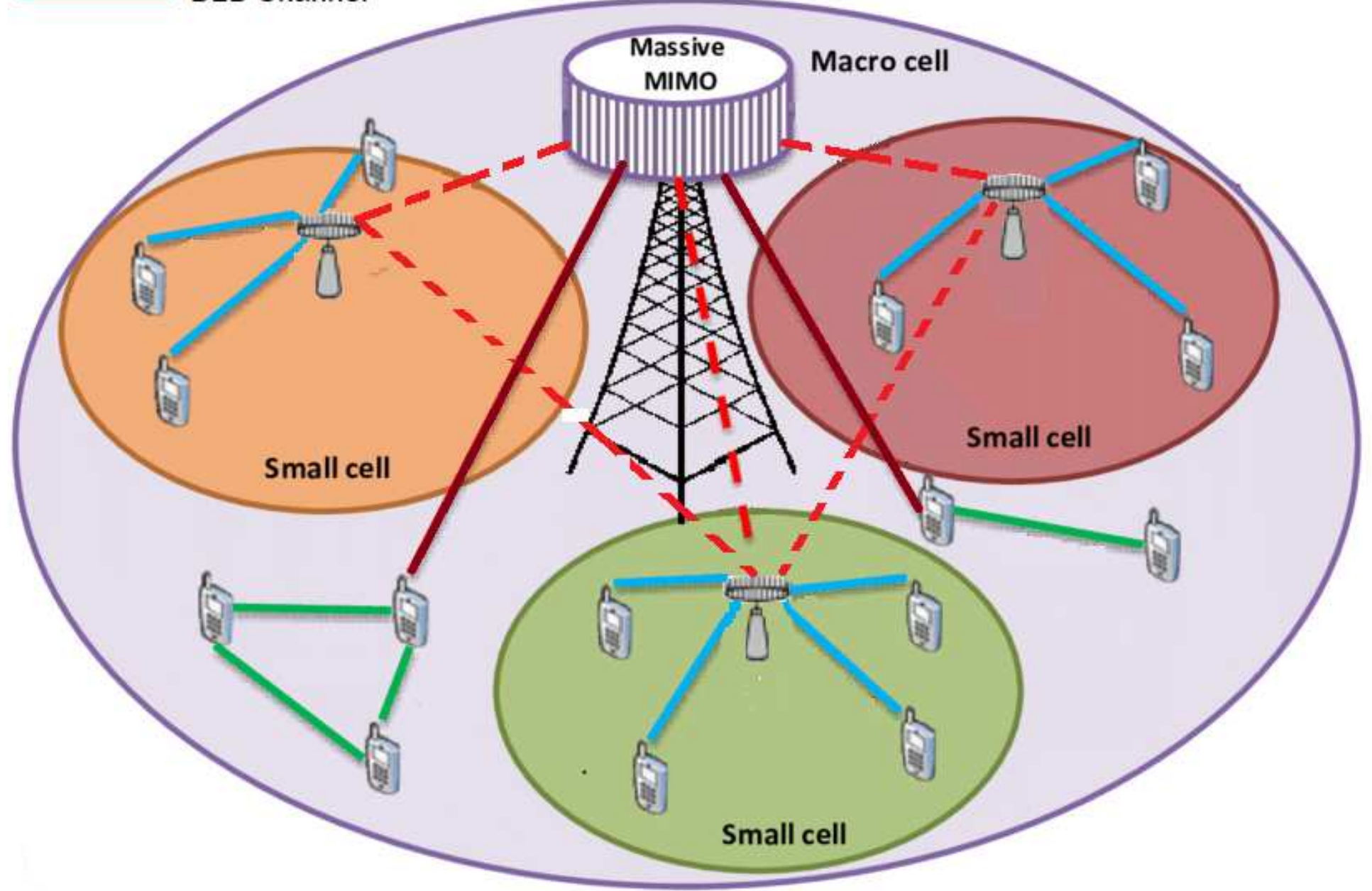

Figure 8

Candidate Network Architecture with mmWave-Massive MIMO for 5G [3]. The arrangement of UDNs, mmWave and massive MIMO communications.

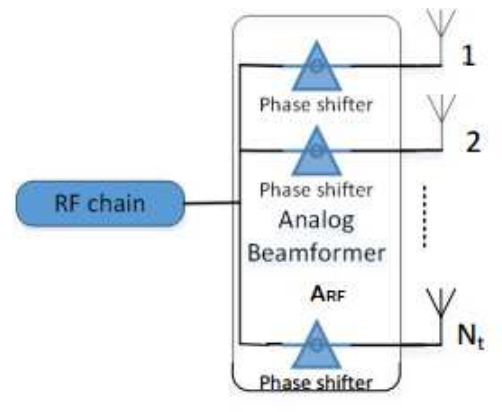

a. Analog Beamforming

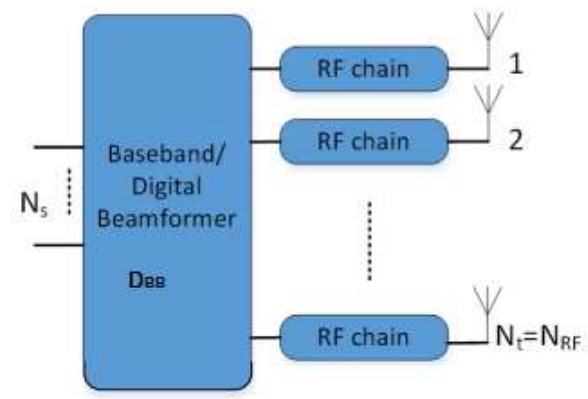

b. Digital Beamforming

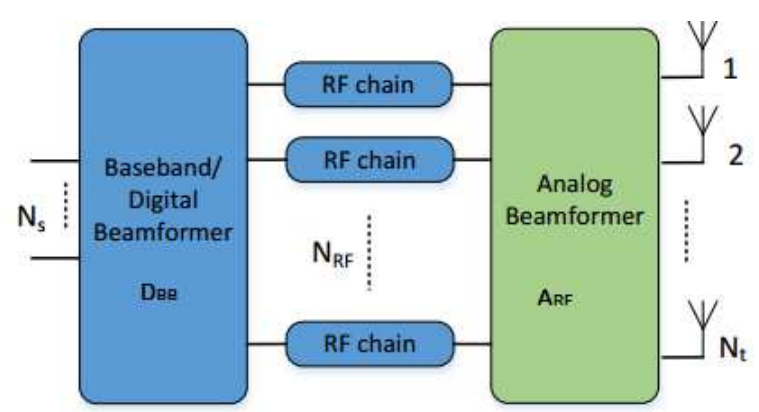

c. Hybrid Beamforming

\section{Figure 9}

Types of Beamforming Architectures [42]. The different types of Beamforming Architec- tures. 


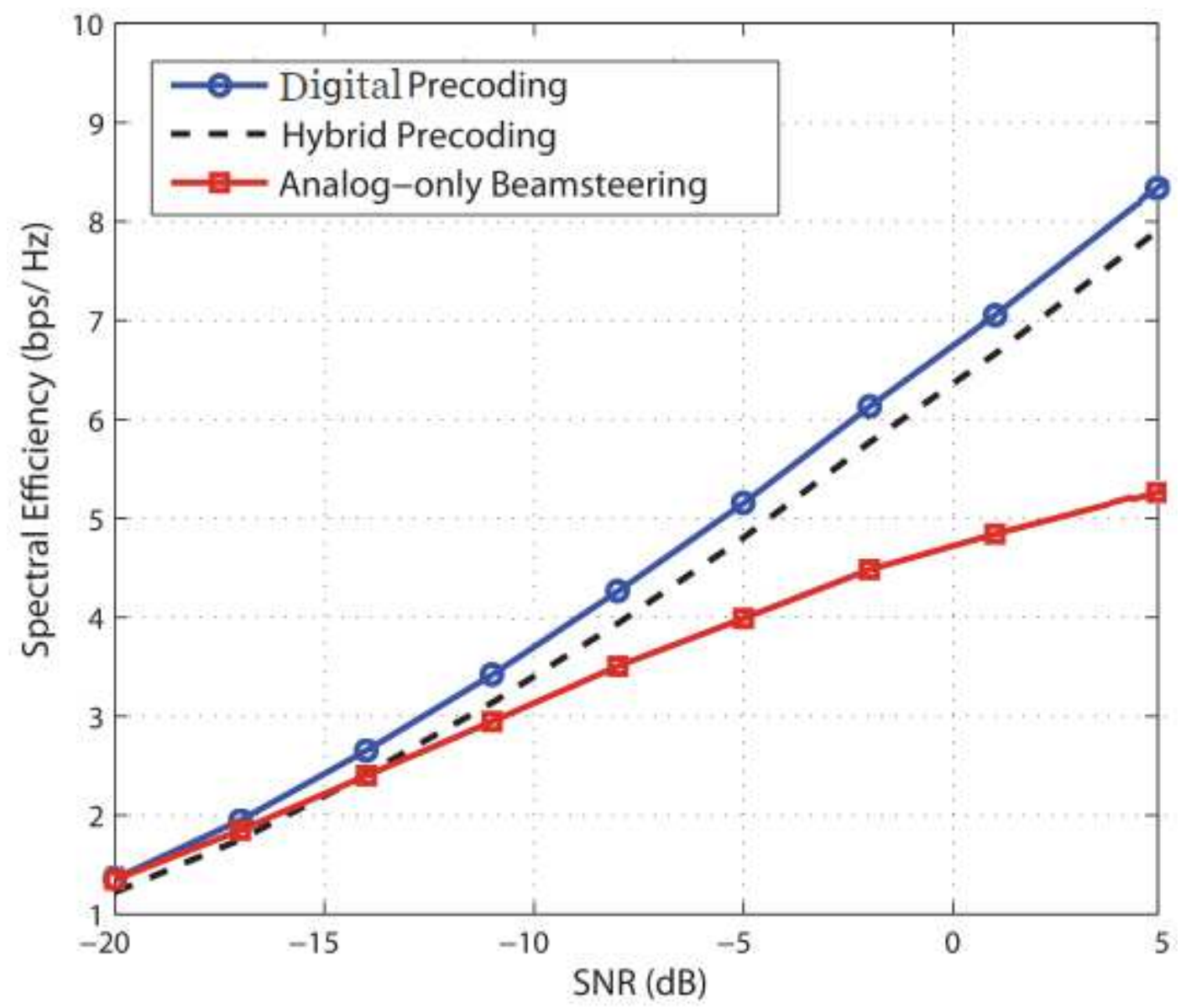

Figure 10

Achievable Rate Versus SNR. The spectral efficiency comparison of the different beam-forming techniques for mmWave-Massive MIMO System.

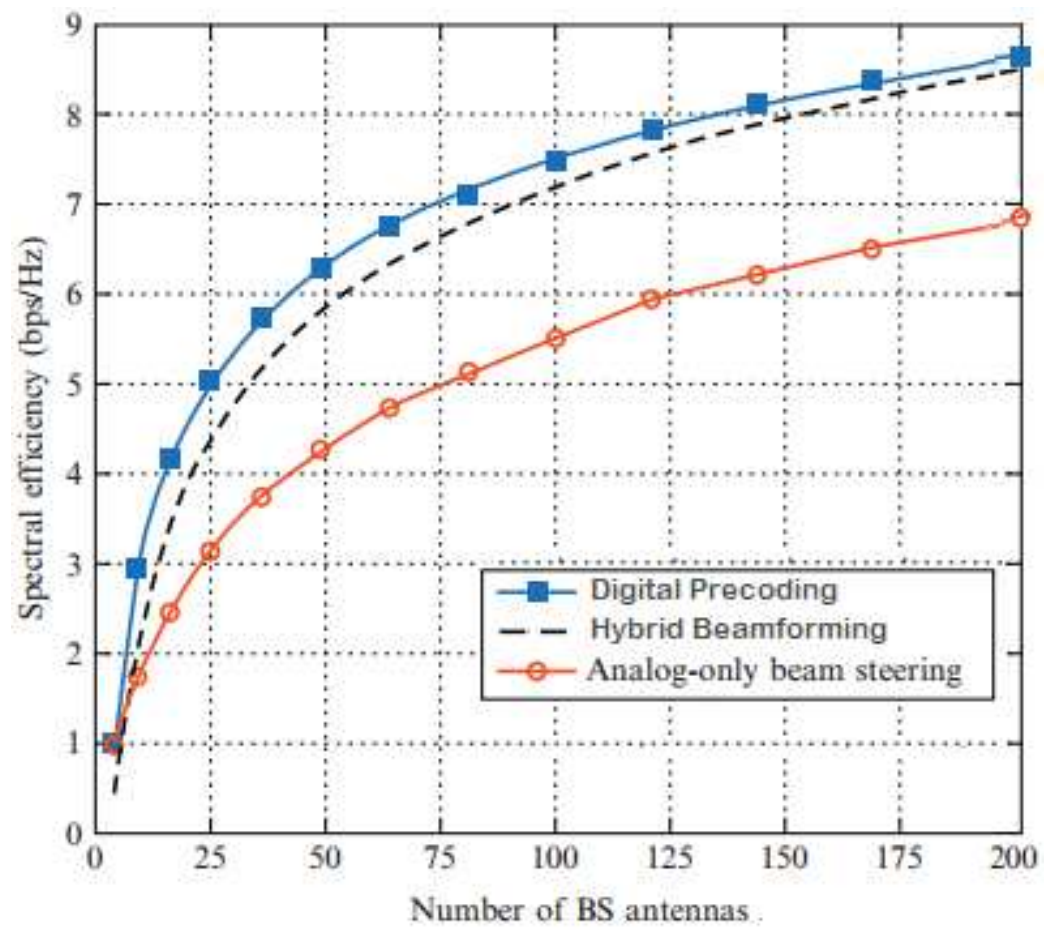


Figure 11

Spectral Efficiency with BS Antennas. The Performance comparison of the different beamforming techniques with resoect to the number of basestation antennas.

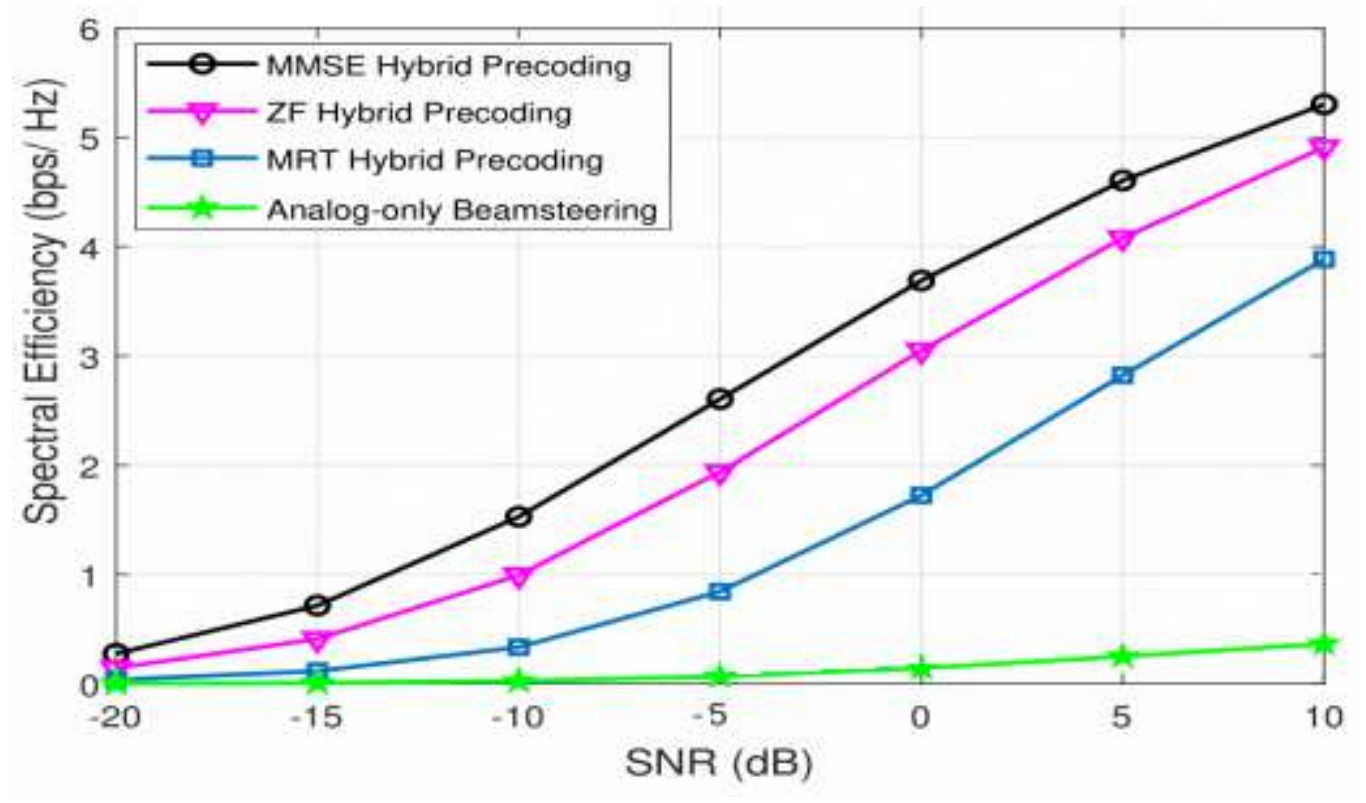

\section{Figure 12}

Spectral Efficiency Versus SNR with different Precoding Schemes. The Spec-tral efficiency of the different types of beamforming techniques are compared with respect to signal to noise ratio.

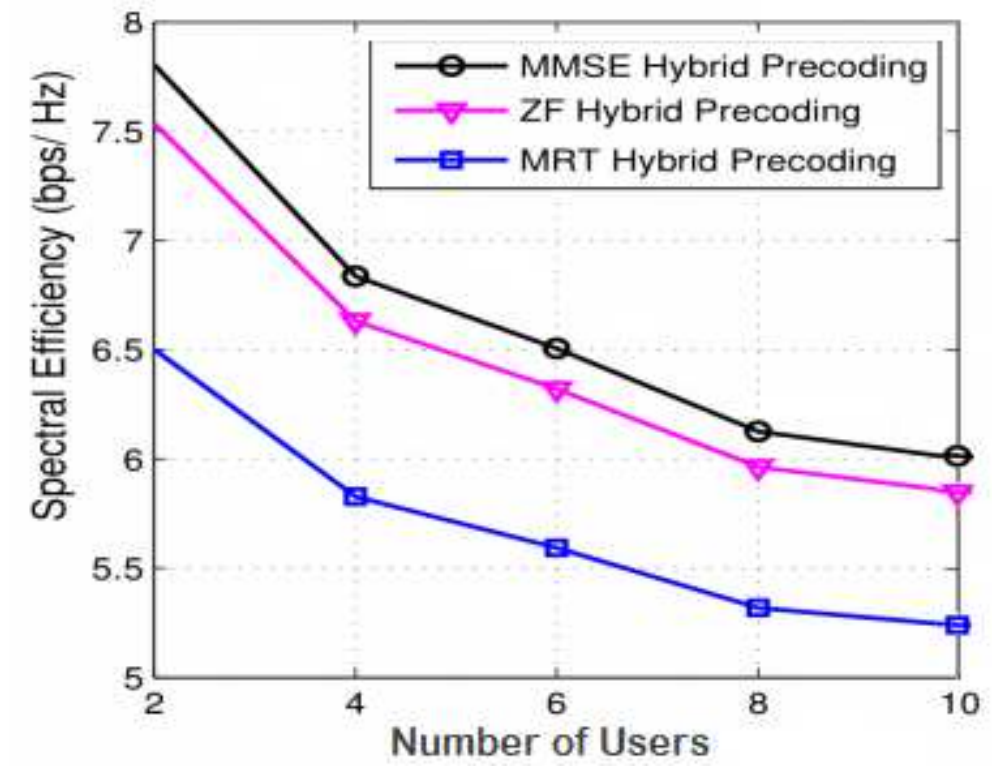

Figure 13

Achievable Rate Versus Number of Users. The Spectral efficiency of the different types of beamforming techniques are compared with respect to the number of users. 


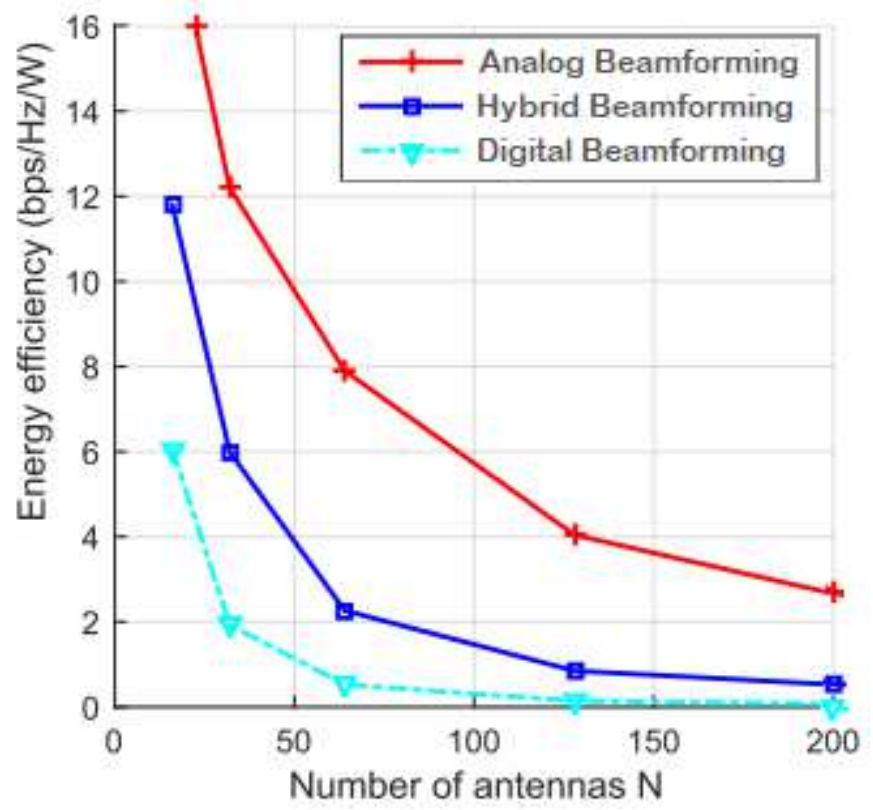

Figure 14

Energy Efficiency Versus Number of Base Station Antennas. The comparison of the different beamforming techniques with number of basesation antennas. The result shows their energy efficiency. 